\title{
Bubble-based stabilized finite element methods for time-dependent convection-diffusion-reaction problems
}

\author{
A. Sendur ${ }^{1, *, \dagger}$ and A. Nesliturk ${ }^{2}$ \\ ${ }^{1}$ Department of Mathematics Education, Alanya Alaaddin Keykubat University, 07490 Antalya, Turkey \\ ${ }^{2}$ Department of Mathematics, Izmir Institute of Technology, 35430 Izmir, Turkey
}

\begin{abstract}
SUMMARY
In this paper, we propose a numerical algorithm for time-dependent convection-diffusion-reaction problems and compare its performance with the well-known numerical methods in the literature. Time discretization is performed by using fractional-step $\theta$-scheme, while an economical form of the residual-free bubble method is used for the space discretization. We compare the proposed algorithm with the classical stabilized finite element methods over several benchmark problems for a wide range of problem configurations. The effect of the order in the sequence of discretization (in time and in space) to the quality of the approximation is also investigated. Numerical experiments show the improvement through the proposed algorithm over the classical methods in either cases. Copyright $@ 2016$ John Wiley \& Sons, Ltd.
\end{abstract}

Received 24 August 2015; Revised 11 January 2016; Accepted 8 February 2016

KEY WORDS: unsteady convection-diffusion-reaction problems; stabilized finite element methods; horizontal/vertical method of lines

\section{INTRODUCTION}

The unsteady convection-diffusion-reaction equations model a variety of phenomenons in physical, chemical and biological sciences [1-6]. Because their analytical solutions are only known under specific circumstances, the numerical simulation of those problems is needed. Further, the derivatives of the analytical solution may be very large in the case of small diffusion, in some small subregions called layers. As the numerical solution of problems containing the layer structures is even a difficult task for problems in steady-state case, the construction of accurate numerical algorithms for such problems in time-dependent cases presents a bigger challenge and less number of work on the subject appears in the literature. [7-11].

There are several approaches for the numerical treatment of the unsteady convection-diffusionreaction problems [12-21]. The common approach in transient problems is to carry out discretizations in two separate stages: spatial discretization by some form of the stabilized finite element methods and temporal integration by time-marching finite difference schemes. Such algorithms enable us to combine various discretization methods for the target problem. Depending on the order of discretization, there are apparently two possibilities: In the first, we achieve full discretization by keeping time continuous, discretizing the equation in space with a stabilized method, and then discretizing the resulting system of ODEs in time with the fractional-step $\theta$-scheme. The overall algorithm is known as vertical method of lines $[10,22]$. The most popular finite element stabilization for the spatial discretization is the streamline upwind Petrov-Galerkin (SUPG) method. The stability of the SUPG method for transient convection-diffusion equations is studied in

\footnotetext{
*Correspondence to: A. Sendur, Department of Mathematics Education, Alanya Alaaddin Keykubat University, 07490 Antalya, Turkey.

${ }^{\dagger}$ E-mail: alisendur@akdeniz.edu.tr 
$[19,23]$ and convection-diffusion-reaction equations in [24]. Variations of the SUPG method such as Galerkin/least-square is also reported in [25]. The advantage of the SUPG-type stabilized methods is their applicability to a wide range of problem configurations and their simplicity in computer implementations; however, the solutions obtained with the SUPG method possess often spurious oscillations (overshoots or undershoots) in narrow regions around sharp layers. These oscillations can even be amplified if high-order finite elements are used [26]. A detailed review of SUPG-related methods can be found in $[27,28]$. Therefore, improved numerical algorithms to produce more accurate and stable results in spatial stabilization are required. The introduction of bubble functions can be seen as further attempt toward that direction. Through their application to convectiondiffusion-reaction equations, it is possible to get better approximations in a wide range of problem configurations [9, 10, 29-34]. However, those functions are defined by a set of local differential equations posed inside each element, which may not be easier to solve than the original one, except that the problem domains are simple element geometries. Taking advantage of that fact gives rise another and more recent spatial discretizations for such problems [35-38]. Those type of methods are based on approximating the bubble functions on a suitably chosen subgrid inside each element, in which the locations of subgrid points are of critical importance, and therefore, they are chosen specially so that fine scale-effect of the exact solution can accurately be represented in the coarse scale numerical approximation. As a first example to those classes, the Link Cutting Bubbles, first introduced in [39] for convection-diffusion-reaction problems and later applied to its time-dependent form in [40], produces improved numerical approximations over the SUPG method and reduces overshoots around the layer. However, the improvement is limited and it is applicable only in one space dimension. On the other hand, the pseudo residual-free bubbles (PRFB) method, which will be the focus point of this work, can be seen as another method in the bubble framework. The PRFB approach was first introduced for the steady-state convection-diffusion problems in [36], and it has been successfully applied to steady-state convection-diffusion-reaction problems in one and two space dimensions [41, 42]. The PRFB method is not only well-suited for the problems at higher space dimensions, but also it is able to produce more improved approximations than the SUPG and link-cutting bubbles (LCB) methods.

The second approach to get a full discretization can be obtained by changing the order of discretization, that is, we discretize the equation first in time with fractional-step $\theta$-scheme, and then discretize the resulting system of steady-state convection-diffusion-reaction equations in space with a stabilized method. That method is also known as horizontal method of lines or Rothe's method [43]. Numerical studies and discussions on similarities between several stabilized finite element methods combined with Rothe's method can be found in [44, 45]. Harari [46] has examined Rothe's method for parabolic problems. Harari and Hauke [47] extended the method for convectiondiffusion-reaction, providing an upper bound on the time step for the onset of spatial instability. In a recent work, the use of Rothe's method with the unusual stabilized finite element method is discussed in [48]. Coupling of various stabilization techniques, including a more recent LCB method, was also examined in [40]. We note that one has to deal with an extra reaction term in the resulting steady-state problem even if the reaction term in the original problem does not exist. Further, the resulting steady-state problem could be reaction dominated. Therefore, it is important to use the stabilized methods that are able to deal with both convection and reaction-dominated regimes [40, 46]. We remark that the methods considered here are all well-suited for reaction-dominated problems.

The goal of the present paper is to discover the potential of the PRFB method as a numerical algorithm for the approximate solution of the unsteady convection-diffusion-reaction problems and compare it with the various numerical approaches regarding two possible ways of designing a fully discretized method. The organization of the paper is as follows: In Section 2, time-dependent convection-diffusion-reaction problem and its variational form is presented . In Sections 3-4, we will give a brief explanation of the numerical methods that we consider for the approximate solution of the unsteady convection-diffusion-reaction problems. Finally, we perform the numerical tests and draw conclusions in Section 5. 


\section{PROBLEM STATEMENT}

The partial differential equation that we want to solve numerically is

$$
u_{t}+\mathcal{L} u=u_{t}-\epsilon \Delta u+\beta \cdot \nabla u+\sigma u=f \text { in } \Omega \times(0, T]
$$

where $u$ is the unknown, $\beta$ is the convection velocity, $\epsilon>0$ is the diffusion coefficient, $\sigma \geqslant 0$ is the reaction coefficient, and $f$ is the source term. The problem domain is bounded and denoted by $\Omega \subset \mathbb{R}^{d}, d=1,2$, and the time interval by $(0, T]$. For simplicity, Equation (1) will be supplied with the homogeneous Dirichlet boundary condition

$$
u=0 \quad \text { on } \partial \Omega, \quad t \in(0, T]
$$

and an initial condition of the form

$$
u=u^{0} \quad \text { in } \Omega, \quad t=0
$$

We assume that $\sigma$ is non-negative piecewise constant, $\beta \in L^{\infty}\left((0, T] ; W^{1, \infty}(\Omega)\right)^{d}, f \in$ $L^{2}\left((0, T] ; L^{2}(\Omega)\right)$, and $u^{0} \in L^{2}(\Omega)$. Under these assumptions, the existence and uniqueness of the solution are guaranteed ([49]).

We start by recalling the abstract variational formulation of the problem (1):

$$
\left\{\begin{array}{l}
\text { Find } u \in L^{2}((0, T] ; V) \cap \mathcal{C}^{0}\left((0, T] ; L^{2}(\Omega)\right) \text { such that } \\
\frac{d}{d t}(u(t), v)+a(u(t), v)=(f(t), v), \quad \forall v \in H_{0}^{1}(\Omega) \\
u(0)=u^{0}
\end{array}\right.
$$

where $V=H_{0}^{1}(\Omega)$. Here, $(\cdot, \cdot)$ denotes the $L^{2}(\Omega)$ inner product, and the bilinear operator is defined by

$$
a(u, v)=\epsilon \int_{\Omega} \nabla u \cdot \nabla v+\int_{\Omega} \beta \cdot \nabla u v+\int_{\Omega} \sigma u v
$$

In the numerical approximation of the problem (4), we use separate numerical algorithms for space and time derivative terms, as that enable us to combine different numerical algorithms. On the other hand, depending on the order of discretization with respect to time and space, there are two possibilities, which are represented by the following labels:

$\mathrm{FD}_{t} \mathrm{FE}_{s}$ : Discretize the problem (1) first in time by using a finite difference scheme and then apply a stabilization technique to the resulting stationary convection-diffusion-reaction equations.

$\mathrm{FE}_{s} \mathrm{FD}_{t}$ : First, perform the spatial discretization to problem (1) by using a stabilized finite element method and then use a finite difference scheme to approximate the solution to the corresponding system of ODE's.

In both cases, we use fractional-step $\theta$-scheme for the time discretization. In space discretization, we will employ the PRFB method and compare it with the well-known SUPG [50] and LCB methods [40]. We further investigate the performance of the PRFB method and other numerical algorithms employed depending on the order of discretization with respect to time and space. In the following sections, we describe the methods that result from these two approaches in details.

\section{DESCRIPTION OF THE FD $T \mathrm{FE}_{S}$ APPROACH}

In this part, we will apply horizontal method of lines (or Rothe's method) to discretize the unsteady convection-diffusion-reaction problem in which time discretization is followed by a discretization in space. 
Let $\mathcal{T}_{h}=\{K\}$ be a regular decomposition of $\Omega$ and $0=t_{0}<t_{1}<\ldots<t_{M}=T$ be a uniform partition of time interval with $\Delta t_{m}=t_{m+1}-t_{m}$. Then, the temporal discretization of (1) leads to the following problem

$$
\frac{u^{n+1}-u^{n}}{\Delta t_{n}}=\theta\left(f^{n+1}-\mathcal{L} u^{n+1}\right)+(1-\theta)\left(f^{n}-\mathcal{L} u^{n}\right) \quad n=0,1, \ldots, M-1
$$

which can be rewritten in the following family of stationary convection-diffusion-reaction problems,

$$
\tilde{\mathcal{L}} u^{n+1}=F^{n} \quad n=0,1, \ldots, M-1
$$

where

$$
\begin{aligned}
\tilde{\mathcal{L}} u(\mathbf{x}, \cdot) & =-\underbrace{\epsilon \theta}_{\tilde{\epsilon}} \Delta u(\mathbf{x}, \cdot)+\underbrace{\beta \theta}_{\tilde{\beta}} \cdot \nabla u(\mathbf{x}, \cdot)+\underbrace{\left(\frac{1}{\Delta t_{n}}+\sigma \theta\right)}_{\tilde{\sigma}} u(\mathbf{x}, \cdot) \\
F^{n} & =\frac{u^{n}}{\Delta t_{n}}-(1-\theta) \mathcal{L} u^{n}+\theta f^{n+1}+(1-\theta) f^{n}
\end{aligned}
$$

Then, for any $v \in H_{0}^{1}(\Omega)$, the corresponding bilinear form can be defined as

$$
\tilde{a}\left(u^{n+1}, v\right)=\left(\frac{u^{n}}{\Delta t_{n}}, v\right)-(1-\theta) a\left(u^{n}, v\right)+\theta\left(f^{n+1}, v\right)+(1-\theta)\left(f^{n}, v\right)
$$

where $n=0,1, \ldots, M-1$ and $\tilde{a}(u, v)=(\tilde{\epsilon} \nabla u, \nabla v)+(\tilde{\beta} \cdot \nabla u, v)+(\tilde{\sigma} u, v)$. On the other hand, to perform the spatial discretization for each $n=0,1, \ldots, M-1$, we use an economical form of the residual-free bubble method $[29,30,34]$ designed for the steady-state problems, whose explicit description is given below.

Observe that, by proceeding in this way, for small $\Delta t_{n}$, we are lead to a family of reactiondominated problems (6) even if the reaction term in the original problem is not dominant. Therefore, a numerical method that produces accurate numerical approximations in reaction-dominated regimes is required in that case.

\subsection{Numerical method}

The principal method employed in space discretization is based on constructing economical approximations to the residual-free bubbles (RFB) functions, called PRFB, by redefining the bubble space $V_{B}$ with those approximate bubbles and use them in the numerical calculations instead of the exact RFB functions. The PRFB method was first designed in the context of the RFB method for the steady-state convection-diffusion problems in [36] and extended to the convection-diffusionreaction problems in [41, 42]. The PRFB functions consist of piecewise linears on a suitably chosen subgrid inside each element. The shape of approximations, which is essentially related with the location of sub-grid points, is crucial to get a good stabilization effect through the numerical method. Therefore, the choice of points in the sub-grid must be fulfilled in a special manner. The location of additional points are determined by means of a minimization process with respect to $L_{1}$ norm in steady-state case. Later, we outline the key steps in the algorithm for spotting sub-grid points for $d=1$ in a typical element $K$. The choice of the sub-grid nodes in 2D case is quite a generalization of the ideas in 1D and we refer to [42] for details.

A typical subinterval $K=\left(x_{k-1}, x_{k}\right), k \in\{1, \ldots, N\}$, on which we approximate the bubble functions, is redefined by adding two points $p_{1}$ and $p_{2}$ with the property that

$$
x_{k-1}<p_{1}<p_{2}<x_{k} .
$$

We denote the length of the $K$ in the subdivision by $h_{K}$. Let us assume that $f$ be a piecewise linear function with respect to the discretization. Consider bubble functions $B_{i},(i=1,2)$ defined by 


$$
\mathcal{L} B_{i}=-\mathcal{L} \psi_{i} \quad \text { in } K, \quad B_{i}=0 \quad \text { on } \partial K, \quad i=1,2
$$

where $\psi_{1}, \psi_{2}$ are the restrictions of the piecewise linear basis functions for $V_{L}$ to $K$, where $V_{L}$ is the space of continuous piecewise linear polynomials on $\mathcal{T}_{h}$. Further, we define $B_{f}$,

$$
\mathcal{L} B_{f}=f \quad \text { in } K, \quad B_{f}=0 \quad \text { on } \partial K .
$$

Solving Equations (10)-(11) may be difficult as much as to solve the original problem in the steadystate case (1). However, using the element geometry and the problem properties, it is possible to construct a cheap, yet efficient approximate bubbles, say $B_{i}^{*}$, over the sub-grid (9), having the same qualitative behavior with its continuous counterpart $B_{i}(i=1,2)$. The construction of such approximate bubble functions $B_{i}^{*}$ is accomplished in the following.

Let $B_{i}^{*}(x)=\alpha_{i} b_{i}(x)$ be the classical Galerkin approximation of $B_{i}$ through (10)

$$
a\left(B_{i}^{*}, b_{i}\right)_{K}=\left(-\mathcal{L} \psi_{i}, b_{i}\right)_{K} \quad, \quad i=1,2
$$

where $b_{i}$ is a piecewise linear function with the following properties

$$
\begin{aligned}
& b_{1}\left(x_{k-1}\right)=b_{1}\left(x_{k}\right)=0, b_{1}\left(p_{1}\right)=1 \\
& b_{2}\left(x_{k-1}\right)=b_{2}\left(x_{k}\right)=0, b_{2}\left(p_{2}\right)=1
\end{aligned}
$$

We must choose $p_{i}$ in a special manner such that the stabilizing effect of bubble function $B_{i}$ is maintained in its discrete counterpart $B_{i}^{*}(i=1,2)$. The main criteria used to determine the locations of the sub-grid nodes is to minimize $L_{1}$ norm of the residual coming out from the bubble Equation (10). In other words, we choose $p_{i}$ such that

$$
J_{i}=\int_{K}\left|\mathcal{L} B_{i}^{*}+\mathcal{L} \psi_{i}\right| d x, \quad i=1,2
$$

is minimum, which yields [41]

$$
p_{1}=\frac{3 \beta+\sqrt{9 \beta^{2}+24 \epsilon \sigma}}{2 \sigma} \quad p_{2}=\frac{-3 \beta+\sqrt{9 \beta^{2}+24 \epsilon \sigma}}{2 \sigma}
$$

However, because the $p_{1}$ and $p_{2}$ are derived in the presence of layers, the locations of sub-grid points must be chosen so that not only the numerical algorithm performs well in other problem regimes, but also it achieves smooth transition between problem regimes. To this end, set

$$
\xi=p_{1}-x_{k-1}, \quad \eta=x_{k}-p_{2}, \quad \delta=p_{2}-p_{1}
$$

Now the explicit description of sub-grid points for each type of problem regime is as follows:

Case: Diffusion-dominated regime: The problem is assumed to be diffusion dominated when $6 \epsilon>$ $\beta h_{K}+\sigma h_{K}^{2} / 9$. In this regime, the stabilization is not needed, and a uniform sub-grid seems to be appropriate. Therefore, we choose $\xi=\eta=\delta=h_{K} / 3$.

Case: Convection-dominated regime: The problem is assumed to be convection dominated if $6 \epsilon \leqslant$ $\beta h_{K}+\sigma h_{K}^{2} / 9$ with $3 \beta \geqslant \sigma h_{K}$. Thus, we take $\eta=\eta_{e}, \delta=\eta$, and $\xi$ is chosen accordingly $\left(\xi=h_{K}-2 \eta\right)$ where

$$
\eta_{e}=\frac{-3 \beta+\sqrt{9 \beta^{2}+24 \epsilon \sigma}}{2 \sigma}
$$

Case: Reaction-dominated regime: The problem is assumed to be reaction-dominated if $6 \epsilon \leqslant$ $\beta h_{K}+\sigma h_{K}^{2} / 9$ and $3 \beta<\sigma h_{K}$. Hence, we take $\eta=\eta_{e}, \xi=\min \left\{h_{K}-2 \eta, \xi_{e}\right\}$ and $\delta$ is chosen accordingly (i.e., $\delta=h_{K}-\eta-\xi$ ), where

$$
\xi_{e}=\frac{3 \beta+\sqrt{9 \beta^{2}+24 \epsilon \sigma}}{2 \sigma}
$$


We now go back to the semi discrete form (8) and apply the PRFB strategy proposed in [41], which yields the following problem

$$
\left\{\begin{array}{l}
\text { Given } u_{h}^{0} \in V_{h}=V_{L} \bigoplus V_{B} \text { find } u_{h}^{n+1} \in V_{h} \text { such that } \forall v_{h} \in V_{h} \\
\tilde{a}\left(u_{h}^{n+1}, v_{h}\right)=\left(u_{h}^{n}, v_{h}\right)-(1-\theta) \Delta t_{n} a\left(u_{h}^{n}, v_{h}\right)+\theta \Delta t_{n}\left(f^{n+1}, v_{h}\right)+(1-\theta) \Delta t_{n}\left(f^{n}, v_{h}\right) \\
n=0,1, \ldots M-1 .
\end{array}\right.
$$

where $V_{B}$ is the discrete bubble space with $V_{B}=\bigoplus_{K} B_{K}$ and $B_{K}=\operatorname{span}\left\{B_{1}^{*}, B_{2}^{*}, B_{f}\right\}$. Here, it is apparent that the bubble space $V_{B}$ should be constructed according to the operator $\tilde{\mathcal{L}}$, rather than $\mathcal{L}$. To test the performance of proposed algorithm, we further compare it with two well-known stabilized methods whose basic features and formulations are summarized in the following remarks.

\section{Remark 1}

The first method that we use in comparisons is the SUPG method, which can be considered as one of the earliest examples to stabilized methods and has been successfully applied to many problems. That approach performs the spatial discretization by adding a consistent term to the standard Galerkin formulation, which, in turn, adds an artificial diffusion in the streamline direction and it reads

$$
\left\{\begin{array}{l}
\text { Given } u_{L}^{0} \in V_{L} \text { find } u_{L}^{n+1} \in V_{L} \text { such that } \forall v_{L} \in V_{L} \\
\tilde{a}\left(u_{L}^{n+1}, v_{L}\right)+\sum_{K \in \mathcal{T}_{h}} \tilde{\tau}_{K}\left(\tilde{\mathcal{L}} u_{L}^{n+1}-F^{n}, \tilde{\beta} \cdot \nabla v_{L},\right)_{K} \\
=\left(u_{L}^{n}, v_{L}\right)-(1-\theta) \Delta t_{n} a\left(u_{L}^{n}, v_{L}\right)+\theta \Delta t_{n}\left(f^{n+1}, v_{L}\right)+(1-\theta) \Delta t_{n}\left(f^{n}, v_{L}\right) \\
n=0,1, \ldots M-1
\end{array}\right.
$$

where $\tilde{\tau}_{K}$ is the intrinsic time parameter. The issue of selecting a proper parameter is essential for the optimal performance of the method and many works in literature have been devoted to this topic $[40,44,51,52]$. We use the following stabilization parameter throughout numerical experiments,

$$
\tilde{\tau}_{K}=\left(\frac{12 \tilde{\epsilon}}{h_{K}^{2}}+\frac{2|\tilde{\beta}|}{h_{K}}+2 \widetilde{\sigma}\right)^{-1}
$$

as three different choices produced similar results in the numerical experiments ( $h_{K}$ being an appropriate measure for the size of the mesh cell $K$ ). We note a further difficulty with the SUPG method at small time steps was reported in $[23,46]$ that it produced spurious oscillations around layers in several numerical experiments.

\section{Remark 2}

The second method that we use in comparisons is proposed by Brezzi et al. in [39]: the LCB method. The underlying idea behind the LCB method is to augment the original mesh by adding suitably chosen two additional points inside each element and to solve the problem on the new, enriched mesh in the context of the plain Galerkin method. The location of the additional nodes added to each element is similar to the ones in the PRFB method and crucial in getting the stabilization. To be precise, define two extra nodes in each element $K$, say $z_{1}$ and $z_{2}$, such that $x_{k-1}<z_{1}<z_{2}<x_{k}$ with

$$
\begin{gathered}
x_{k}-z_{2}=\min \left\{\frac{h_{K}}{3}, \frac{-3 \beta+\sqrt{9 \beta^{2}+24 \epsilon \sigma}}{2 \sigma}\right\} \\
z_{1}-x_{k-1}=\min \left\{h_{K}-2\left(x_{2}-z_{2}\right), \frac{3 \beta+\sqrt{9 \beta^{2}+24 \epsilon \sigma}}{2 \sigma}\right\}
\end{gathered}
$$

and obtain the enriched grid, say $\mathcal{K}_{h}$, having both the original grid nodes and the added subgrid nodes all together. The LCB operates on $\mathcal{K}_{h}$ as a plain Galerkin method and it reads 
$\left\{\begin{array}{l}\text { Given } u_{E}^{0} \in V_{E} \text { find } u_{E}^{n+1} \in V_{E} \text { such that } \forall v_{E} \in V_{E} \\ \tilde{a}\left(u_{E}^{n+1}, v_{E}\right)=\left(u_{E}^{n}, v_{E}\right)-(1-\theta) \Delta t_{n} a\left(u_{E}^{n}, v_{E}\right)+\theta \Delta t_{n}\left(f^{n+1}, v_{E}\right)+(1-\theta) \Delta t_{n}\left(f^{n}, v_{E}\right) \\ n=0,1, \ldots M-1\end{array}\right.$

where $V_{E}$ is the space of continuous piecewise linear polynomials on augmented mesh $\mathcal{K}_{h}$. We refer to [39] for details. We note that the LCB discretization of (8) is obtained by constructing the subgrid with respect to the operator $\tilde{\mathcal{L}}$, that is, use (19)-(20) with modified parameters $\tilde{\epsilon}, \tilde{\beta}$, $\tilde{\sigma}$. Although the LCB method produces more improved numerical approximations compared with the SUPG, it is not able to eliminate overshoots around the layer at all. Further, it is observed that numerical results obtained through the LCB method is dispersive in some benchmark problems.

\section{DESCRIPTION OF THE FE $\mathrm{FD}_{T}$ APPROACH}

Although we use the same numerical methods presented in the previous section for both time and space terms, we change their order of discretization and test the effect of that change into the quality of the approximation for the methods of interest. This time, we will apply vertical method of lines to discretize the unsteady convection-diffusion-reaction problem in which space discretization is followed by a discretization in time. We note that such an approach allows the application of modern stationary finite element codes to time-dependent problems with few modifications only.

Now, if we discretize the problem (1) first in space by the PRFB method, we are lead to the following semidiscrete finite element method

$$
\left\{\begin{array}{l}
\text { Find } u_{h}:[0, T] \rightarrow V_{h}=V_{L} \oplus V_{B} \text { such that } \forall v_{h} \in V_{h} \\
\frac{d}{d t}\left(u_{h}(t), v_{h}\right)=\underbrace{-a\left(u_{h}(t), v_{h}\right)+\left(f, v_{h}\right)}_{W(t)}
\end{array}\right.
$$

The resulting system of ODEs can be integrated in time by using fractional-step $\theta$-scheme to get

$$
\left(\frac{u_{h}^{n+1}-u_{h}^{n}}{\Delta t_{n}}, v_{h}\right)=\theta W^{n+1}+(1-\theta) W^{n} \quad n=0,1, \ldots, M-1
$$

Rearranging the terms, we get the following fully discretized problem:

$$
\left\{\begin{aligned}
\text { For } n=0,1, \ldots, M-1 \text { find } u_{h}^{n+1} & \in V_{h} \text { such that } \forall v_{h} \in V_{h} \\
\left(u_{h}^{n+1}, v_{h}\right)+\theta \Delta t_{n} a\left(u_{h}^{n+1}, v_{h}\right)= & \left(u_{h}^{n}, v_{h}\right)-(1-\theta) \Delta t_{n} a\left(u_{h}^{n}, v_{h}\right) \\
& +\theta \Delta t_{n}\left(f^{n+1}, v_{h}\right)+(1-\theta) \Delta t_{n}\left(f^{n}, v_{h}\right)
\end{aligned}\right.
$$

Note that construction of the bubble space $V_{B}$ and the choice of internal nodes $\xi$ and $\eta$ are accomplished with respect to the operator $\mathcal{L}$. The outline of other two space discretization methods is similar and given in the following remarks. Their numerical comparison are made in coming section.

\section{Remark 3}

If we employ the SUPG method in space discretization and follow the steps previously mentioned, we are lead to the following problem:

$$
\left\{\begin{array}{l}
\text { Find } u_{L}:[0, T] \rightarrow V_{L} \text { such that } \forall v_{L} \in V_{L} \\
\frac{d}{d t}\left(u_{L}(t), v_{L}\right)=\underbrace{-a\left(u_{L}(t), v_{L}\right)-\sum_{K \in \mathcal{T}_{h}} \tau_{K}\left(u_{t}+\mathcal{L} u_{L}(t)-f, \beta \cdot \nabla v_{L},\right)_{K}+\left(f(t), v_{L}\right)}_{G(t)}
\end{array}\right.
$$


where the integration in time of the aforementioned system of ODEs with fractional-step $\theta$-scheme leads to the following fully discrete problem,

$$
\left\{\begin{array}{l}
\text { For } n=0,1, \ldots, M-1 \text { find } u_{L}^{n+1} \in V_{h} \text { such that } \forall v_{L} \in V_{L} \\
\left(u_{L}^{n+1}, v_{L}\right)+\theta \Delta t_{n} a\left(u_{L}^{n+1}, v_{L}\right)+\theta \Delta t_{n} \sum_{K \in \mathcal{T}_{h}} \tau_{K} \quad\left(\mathcal{L} u_{L}^{n+1}, \beta \cdot \nabla v_{L}\right)_{K} \\
+\sum_{K \in \mathcal{T}_{h}} \tau_{K}\left(u_{L}^{n+1}, \beta \cdot \nabla v_{L}\right)_{K}=\left(u_{L}^{n}, v_{L}\right)+\sum_{K \in \mathcal{T}_{h}} \tau_{K}\left(u_{L}^{n}, \beta \cdot \nabla v_{L}\right)_{K} \\
\theta \Delta t_{n} \sum_{K \in \mathcal{T}_{h}} \tau_{K} \quad\left(f^{n+1}, \beta \cdot \nabla v_{L}\right)_{K}+\theta \Delta t_{n}\left(f^{n+1}, v_{L}\right)-(1-\theta) \Delta t_{n} a\left(u_{L}^{n}, v_{L}\right) \\
-(1-\theta) \Delta t_{n} \sum_{K \in \mathcal{T}_{h}} \tau_{K} \quad\left(\mathcal{L} u_{L}^{n}-f^{n}, \beta \cdot \nabla v_{L}\right)_{K}+(1-\theta) \Delta t_{n}\left(f^{n}, v_{L}\right)
\end{array}\right.
$$

where the algorithmic parameter $\tau_{K}$ is set to be

$$
\tau_{K}=\left(\frac{12 \epsilon}{h_{K}^{2}}+\frac{2|\beta|}{h_{K}}+2 \sigma\right)^{-1}
$$

\section{Remark 4}

With regards to the LCB strategy, performing the spatial discretization according to (1), we are lead to the following problem (in time-continuous form),

$$
\left\{\begin{array}{l}
\text { Find } u_{E}:[0, T] \rightarrow V_{E} \text { such that } \forall v_{E} \in V_{E} \\
\frac{d}{d t}\left(u_{E}(t), v_{E}\right)+a\left(u_{E}(t), v_{E}\right)=\left(f, v_{E}\right)
\end{array}\right.
$$

Integration in time of the aforementioned system of ODEs with fractional-step $\theta$-scheme leads to the following fully discrete problem,

$$
\left\{\begin{aligned}
\text { For } n=0,1, \ldots, M-1 \text { find } u_{E}^{n+1} \in V_{E} \text { such that } \forall v_{E} \in V_{E} \\
\begin{array}{rl}
\left(u_{E}^{n+1}, v_{E}\right)+\theta \Delta t_{n} a\left(u_{E}^{n+1}, v_{E}\right)= & \left(u_{E}^{n}, v_{E}\right)-(1-\theta) \Delta t_{n} a\left(u_{h}^{n}, v_{E}\right) \\
& +\theta \Delta t_{n}\left(f^{n+1}, v_{E}\right)+(1-\theta) \Delta t_{n}\left(f^{n}, v_{E}\right)
\end{array}
\end{aligned}\right.
$$

Note that the subgrid is constructed with respect to the operator $\mathcal{L}$ and we take them as in (19)-(20).

\section{NUMERICAL RESULTS}

In this section, the methods described in Sections 3-4 are compared for the unsteady convectiondiffusion-reaction problems. This comparison is carried out by computing the numerical solutions, especially in the interesting case of small diffusion on a set of test problems. We further include a nonlinear example, the Burgers' equation, which is a fundamental partial differential equation occuring in various areas of applied mathematics. We also observe that three stabilized methods produce the least error at different Courant-numbers $\left(C F L=\frac{\Delta t|\beta|}{h}\right)$, and we remark that we use the best CFL value for the corresponding stabilized method. This will allow us to discuss the ability of the numerical methods for reducing the spurious oscillations at their best. A comparative study for those CFL values can be found in [40, 53, 54].

5.0.1. Experiment 1. We start the numerical experiments with a test problem whose initial condition is presented in Figure 1 and can be defined as,

$$
u(x, 0)=x
$$

and the boundary condition as $u(0, t)=0, u(1, t)=1$. We take a uniform partition of $\Omega$ into subintervals of length $h=1 / 20,1 / 40$. In Figures 2-7, we set the value of the diffusion coefficient to be $\epsilon=10^{-6}$, the convective field $\beta=1$, the external force $f=0$, the final time $T=0.5$, and plot the solutions for different values of reaction $\sigma=10^{-4}, 1,10$. 


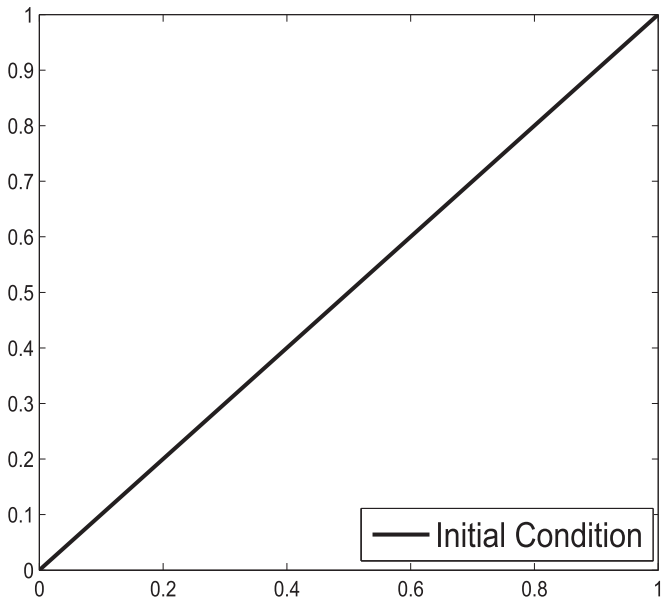

Figure 1. Experiment 1: initial condition.

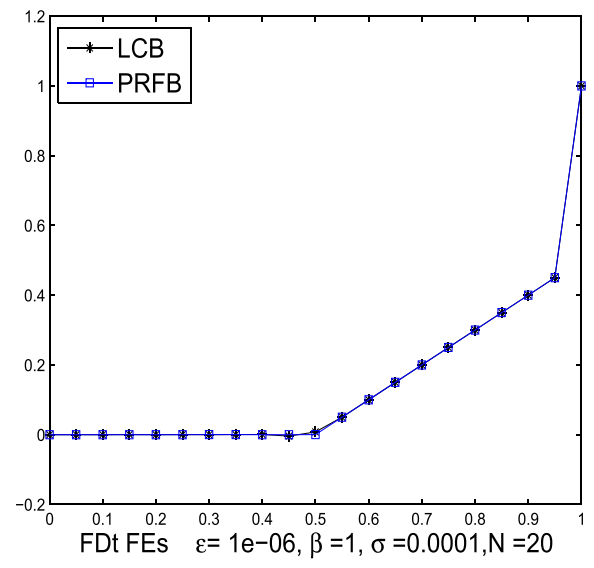

(a)

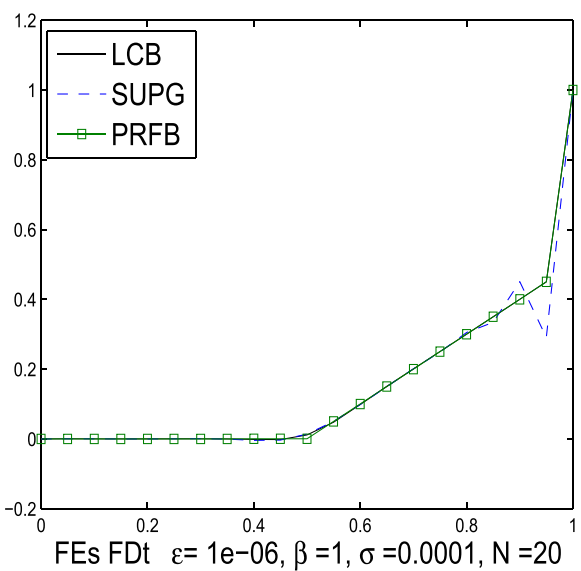

(b)

Figure 2. The numerical solutions for $\sigma=10^{-4}, \theta=1 / 2$ and $h=1 / 20$.

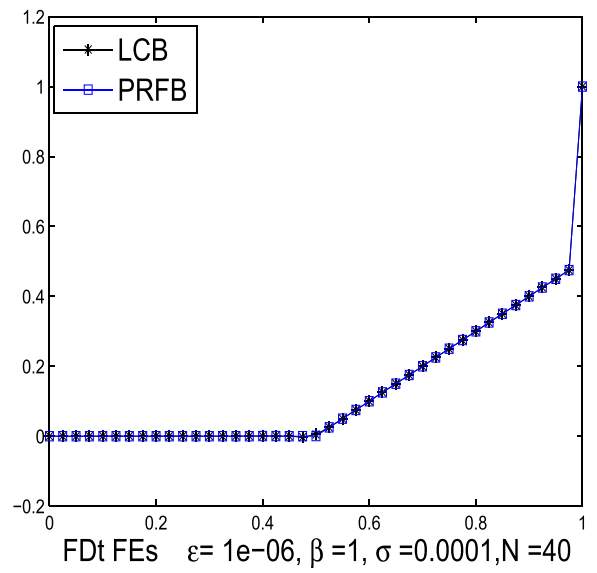

(a)

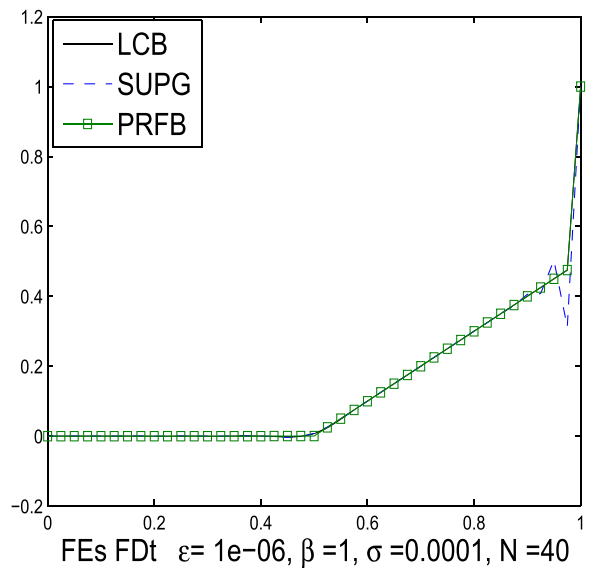

(b)

Figure 3. The numerical solutions for $\sigma=10^{-4}, \theta=1 / 2$ and $h=1 / 40$. 


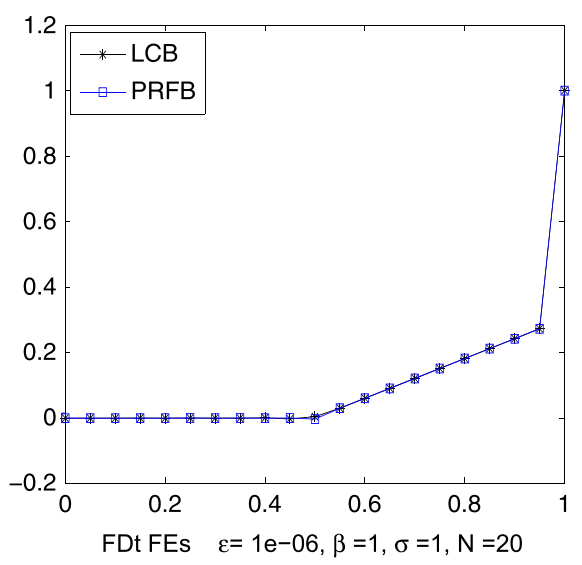

(a)

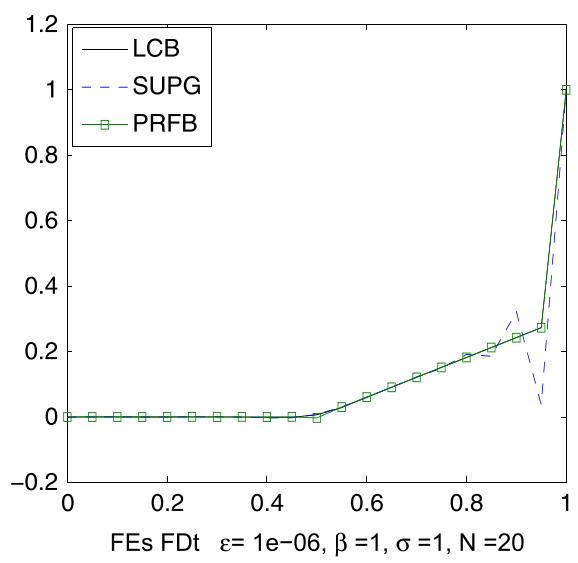

(b)

Figure 4. The numerical solutions for $\sigma=1, \theta=1 / 2$ and $h=1 / 20$.

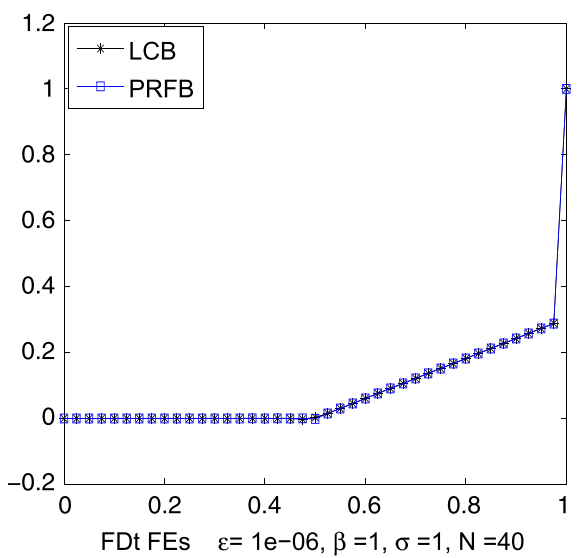

(a)

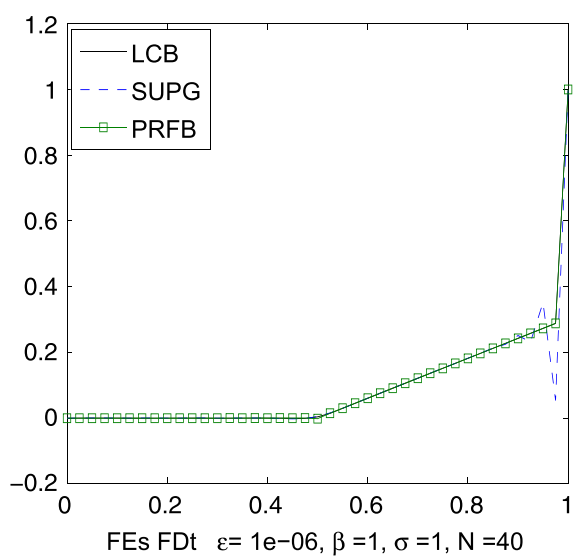

(b)

Figure 5. The numerical solutions for $\sigma=1, \theta=1 / 2$ and $h=1 / 40$.

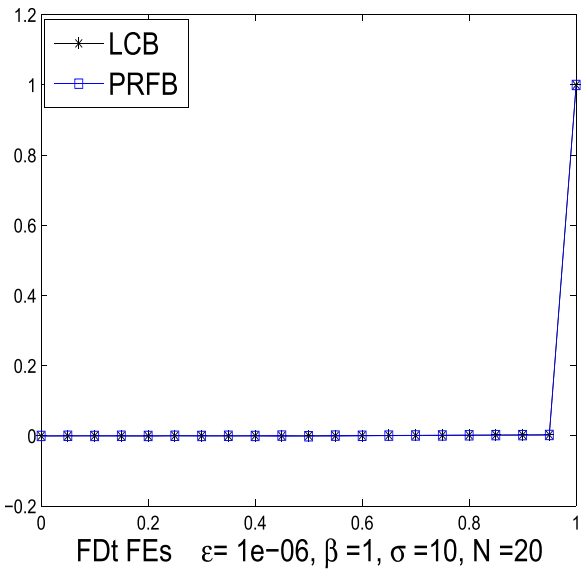

(a)

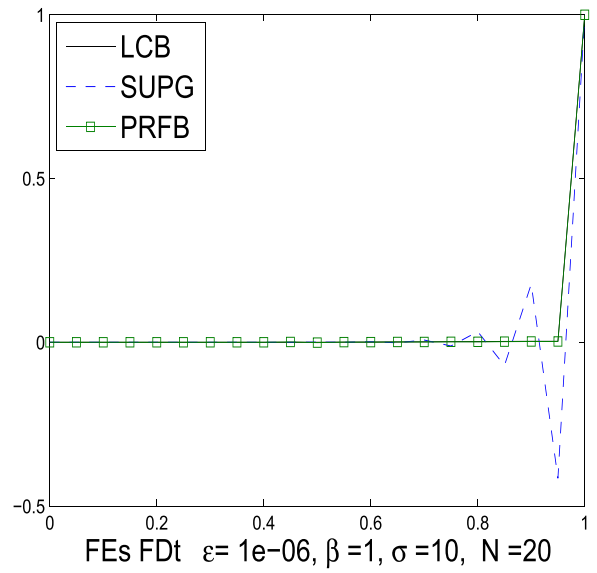

(b)

Figure 6. The numerical solutions for $\sigma=10, \theta=1 / 2$ and $h=1 / 20$. 


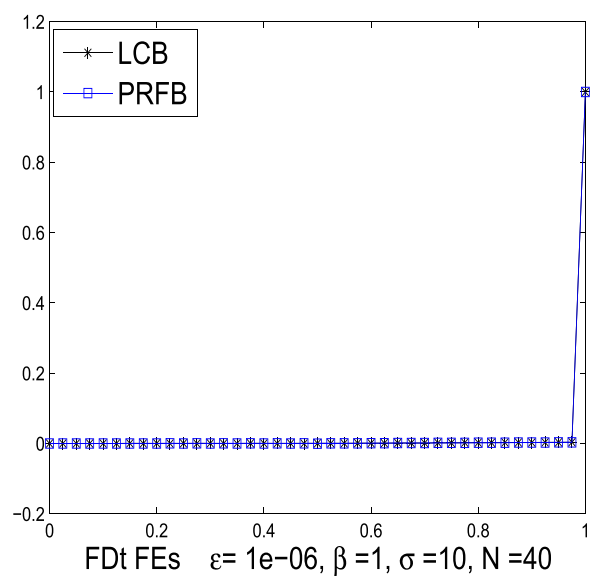

(a)

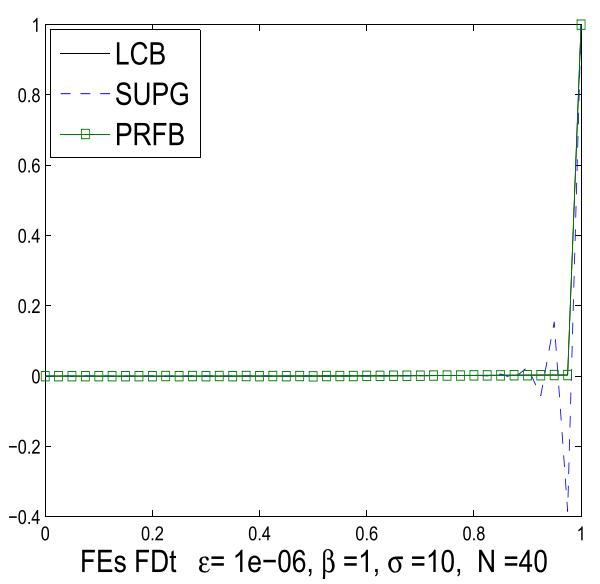

(b)

Figure 7. The numerical solutions for $\sigma=10, \theta=1 / 2$ and $h=1 / 40$.

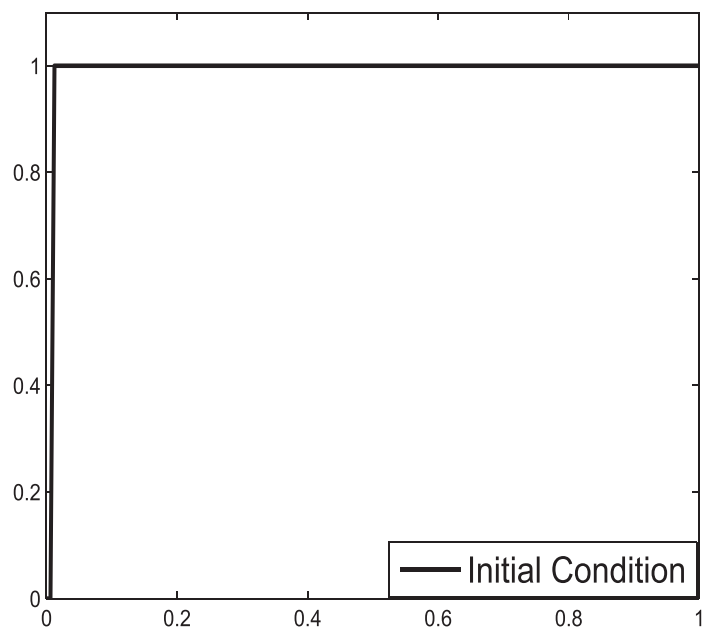

Figure 8. Transport of a boundary layer: initial condition.

Here, we did not display the results for the SUPG method combined with $\mathrm{FD}_{t} \mathrm{FE}_{s}$ approach because it produced oscillations that spread throughout the computational domain. The numerical solutions obtained with the PRFB and LCB methods are in excellent agreement for the whole problem parameters, while the approximations generated by the SUPG method possess spurious oscillations in layer regions.

5.0.2. Experiment 2: transport of a boundary layer. Now we consider the convection of a discontinuous initial data at unit speed $[48,55]$. The discontinuity occurs over one element and is initially located at position $x=0.01$ of the domain $[0,1]$ (Figure 8 ). The discontinuity is given as

$$
u(x, 0)= \begin{cases}1, & \text { if } x>0.01 \\ 0, & \text { if } x \leqslant 0.01\end{cases}
$$

and the boundary condition as $u(0, t)=0, u(1, t)=1$. We take a uniform partition of $\Omega$ into subintervals of length $h=1 / 20,1 / 40,1 / 80$. In Figures 9-14, we set the value of the diffusion coefficient to be $\epsilon=10^{-6}$, the convective field $\beta=1$, the external force $f=0$, the final time $T=0.2$, and plot the solutions for different values of reaction $\sigma=10^{-4}, 1$. 


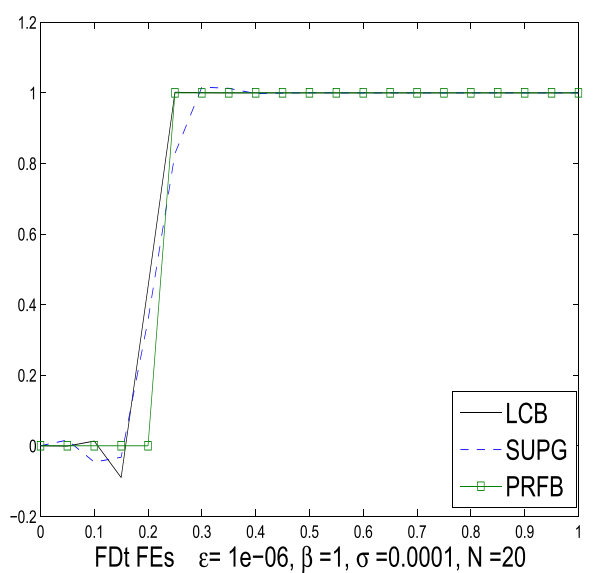

(a)

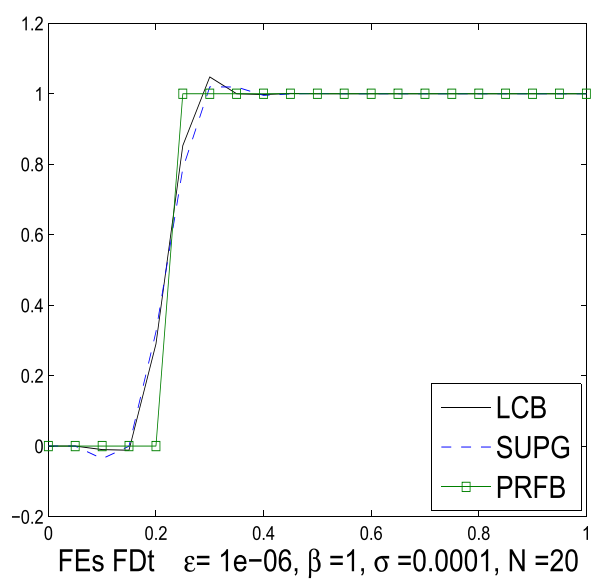

(b)

Figure 9. The numerical solutions for $\sigma=10^{-4}, \theta=1 / 2$, and $h=1 / 20$.

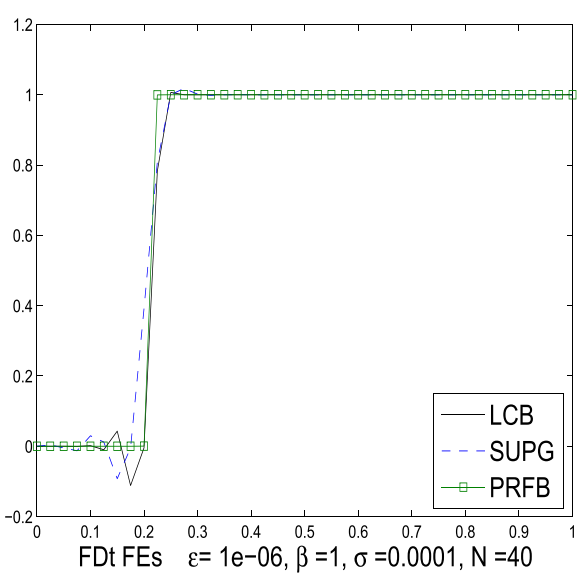

(a)

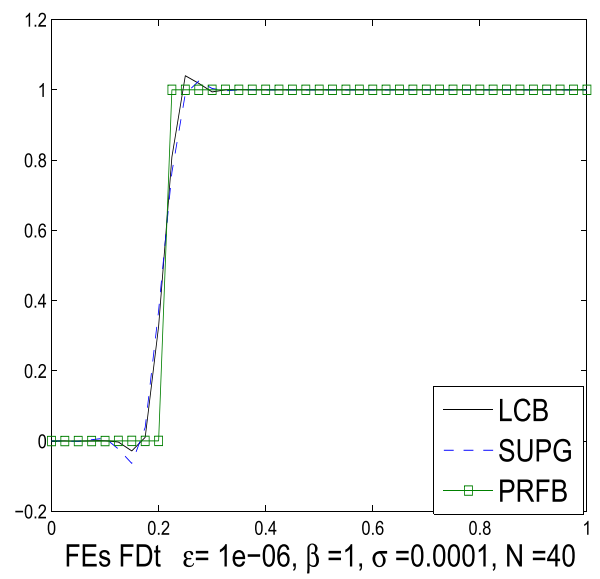

(b)

Figure 10. The numerical solutions for $\sigma=10^{-4}, \theta=1 / 2$, and $h=1 / 40$.

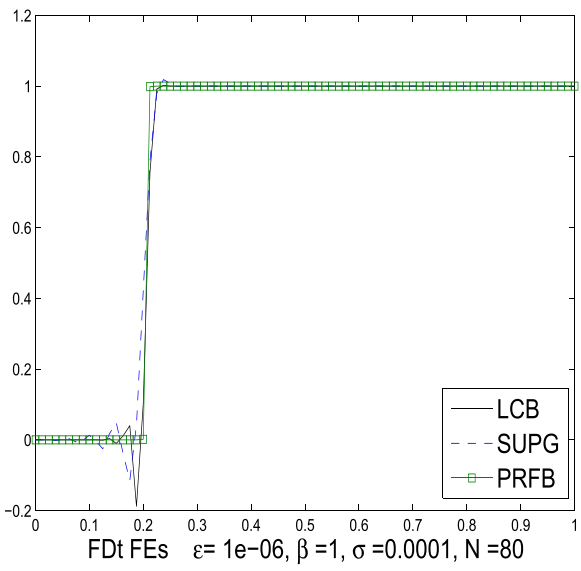

(a)

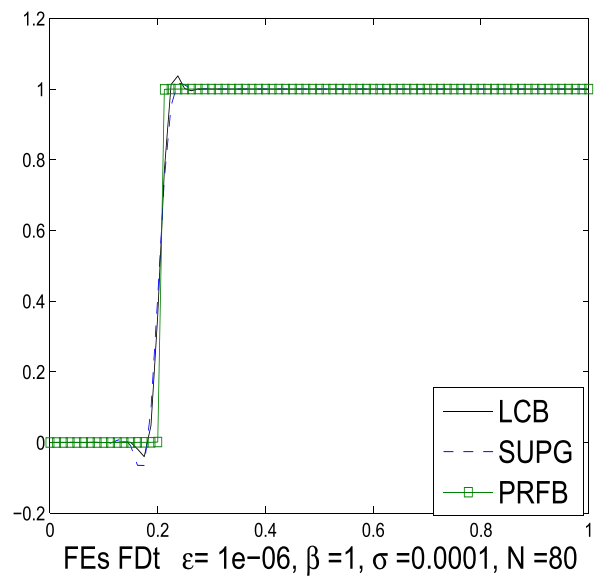

(b)

Figure 11. The numerical solutions for $\sigma=10^{-4}, \theta=1 / 2$, and $h=1 / 80$. 


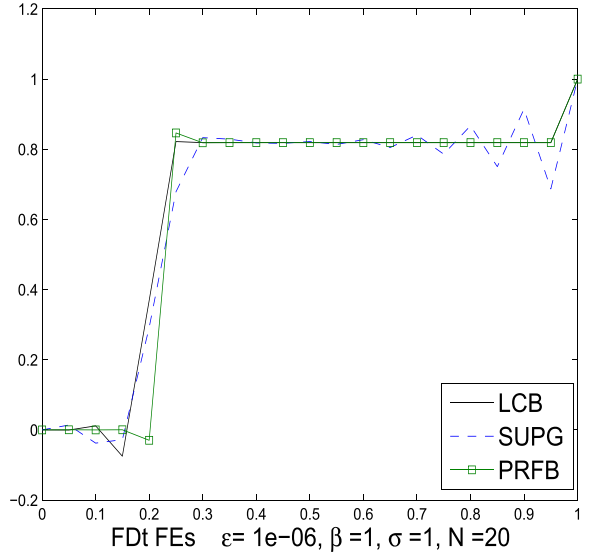

(a)

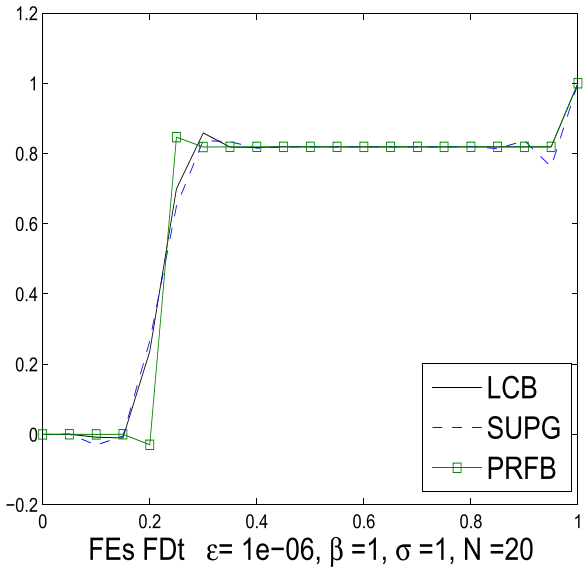

(b)

Figure 12. The numerical solutions for $\sigma=1, \theta=1 / 2$, and $h=1 / 20$.

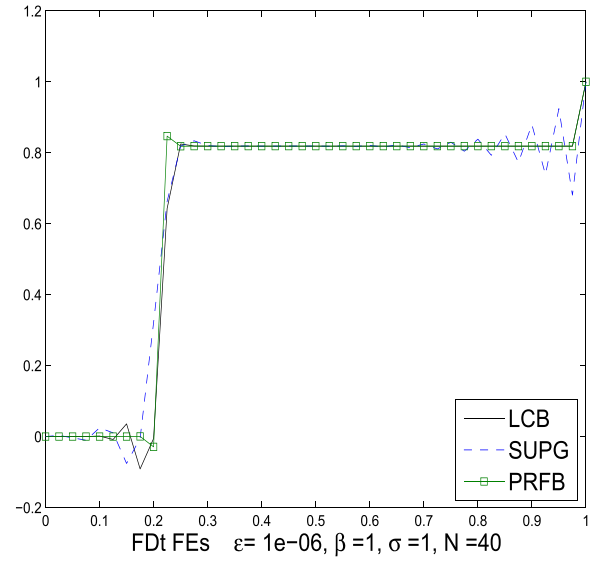

(a)

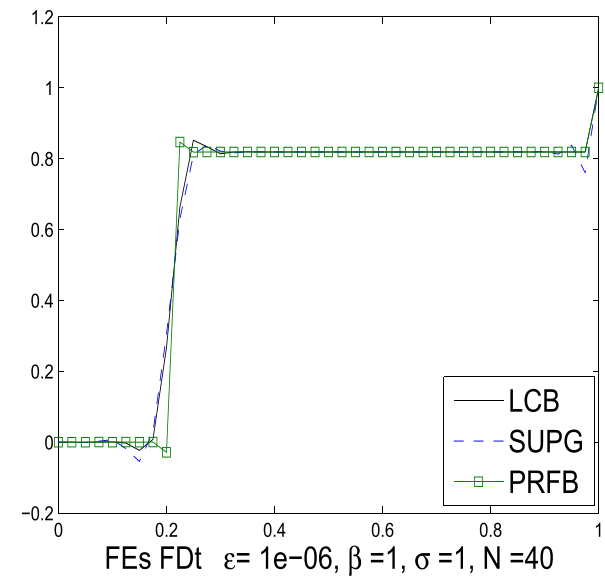

(b)

Figure 13. The numerical solutions for $\sigma=1, \theta=1 / 2$, and $h=1 / 40$.

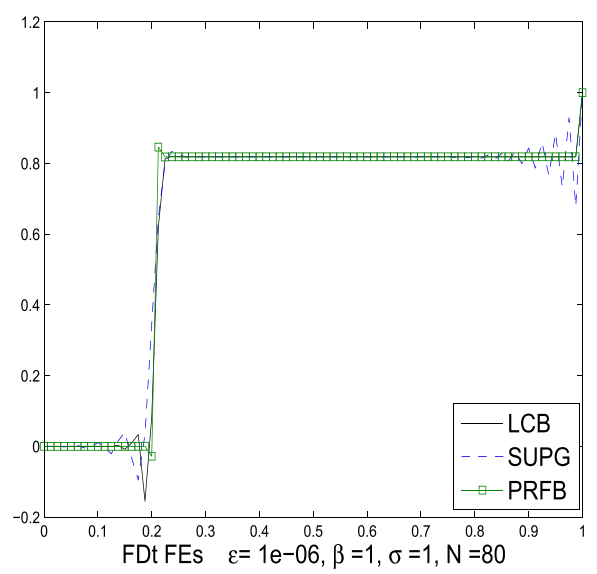

(a)

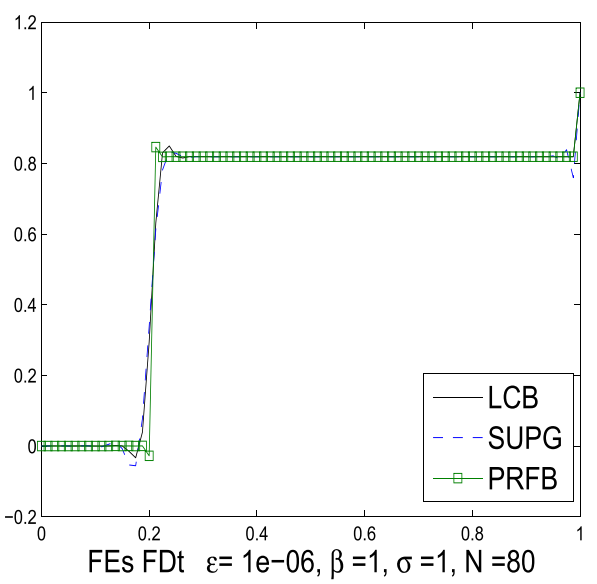

(b)

Figure 14. The numerical solutions for $\sigma=1, \theta=1 / 2$, and $h=1 / 80$. 


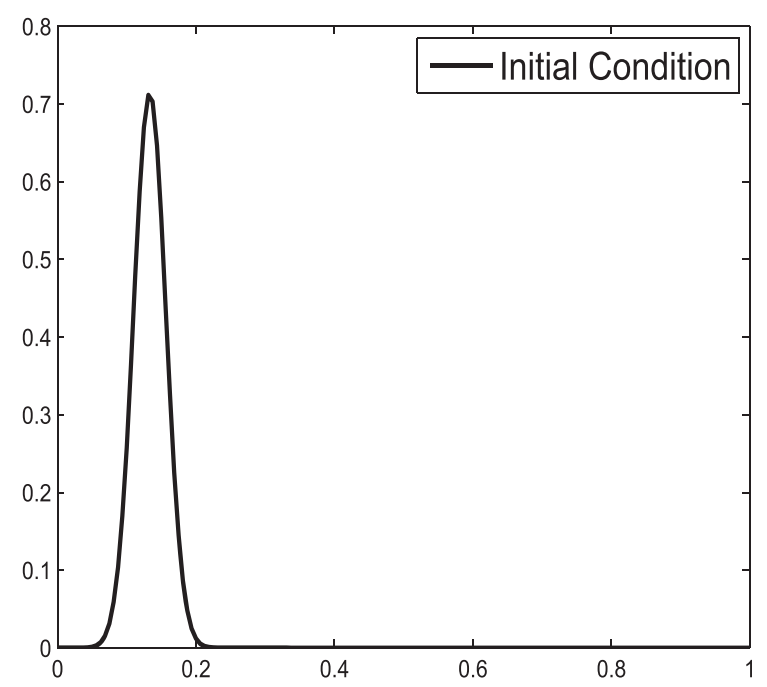

Figure 15. Gaussian hill: initial condition.

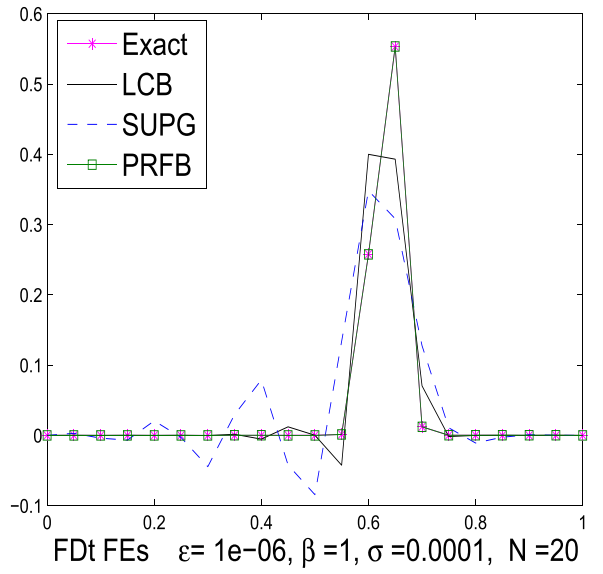

(a)

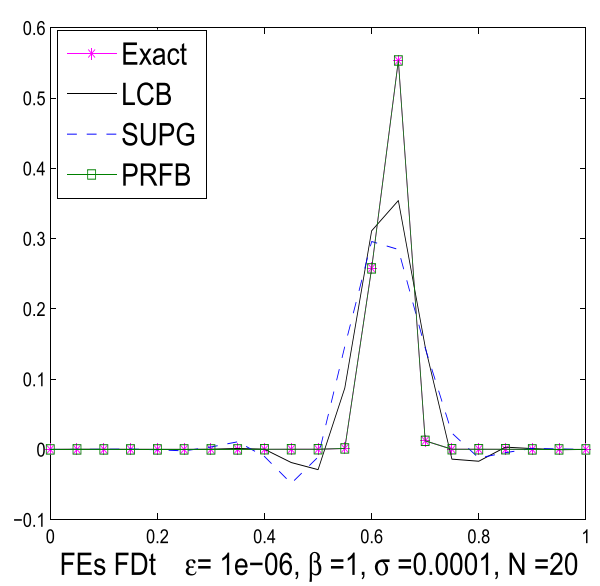

(b)

Figure 16. The numerical solutions for $\sigma=10^{-4}, \theta=1 / 2$, and $h=1 / 20$.

The numerical solutions obtained with the SUPG method show that when using the $\mathrm{FD}_{t} \mathrm{FE}_{s}$ approach, the diffusion added in the streamline direction by the classical stabilization techniques, is not enough to eliminate the spurious oscillations, and in the $\mathrm{FE}_{s} \mathrm{FD}_{t}$ approach, the oscillations are not completely removed from the approximation. The LCB method performs better with $\mathrm{FD}_{t} \mathrm{FE}_{s}$ than with the other approach. The PRFB solution captures the characteristic features of the exact solution in both $\mathrm{FD}_{t} \mathrm{FE}_{s}$ and $\mathrm{FE}_{s} \mathrm{FD}_{t}$ approaches even on coarse meshes.

5.0.3. Experiment 3: transport of Gaussian hill. In this problem, taken from Donea and Huerta [55], a Gaussian distribution profile is convected over 1D domain with the initial condition (Figure 15)

$$
u(x, 0)=\frac{5}{7} e^{-\left(x-x_{0}\right)^{2} / l_{2}} \quad \text { with } \quad x_{0}=2 / 15 \quad \text { and } \quad l_{2}=(7 \sqrt{2} / 300)^{2}
$$

and the boundary condition as $u(0, t)=u(1, t)=0$. We take a uniform partition of $\Omega$ into subintervals of length $h=1 / 20,1 / 40,1 / 80,1 / 160$. In Figures 16-23, we set the value of the diffusion coefficient to be $\epsilon=10^{-6}$, the convective field $\beta=1$, the external force $f=0$, the final time $T=0.5$, and plot the solutions for different values of reaction $\sigma=10^{-4}, 1,10$. 


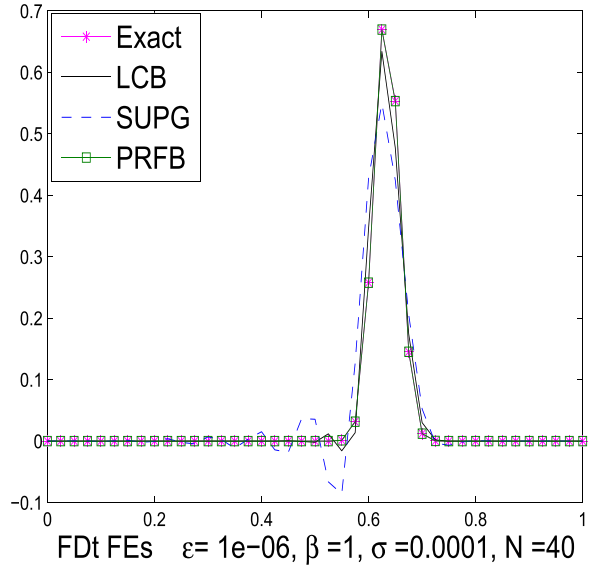

(a)

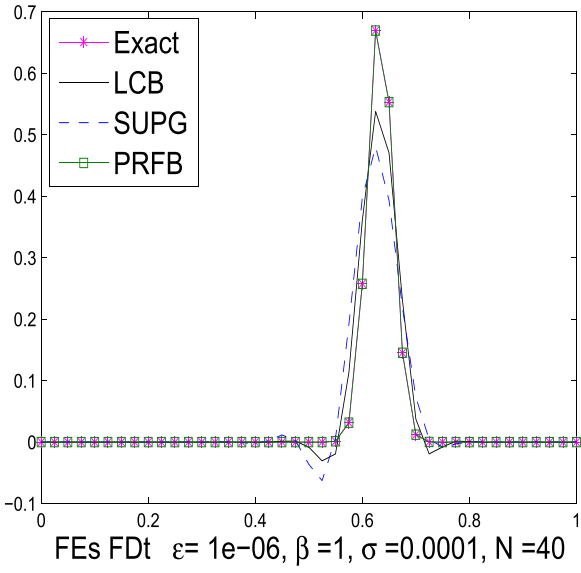

(b)

Figure 17. The numerical solutions for $\sigma=10^{-4}, \theta=1 / 2$, and $h=1 / 40$.

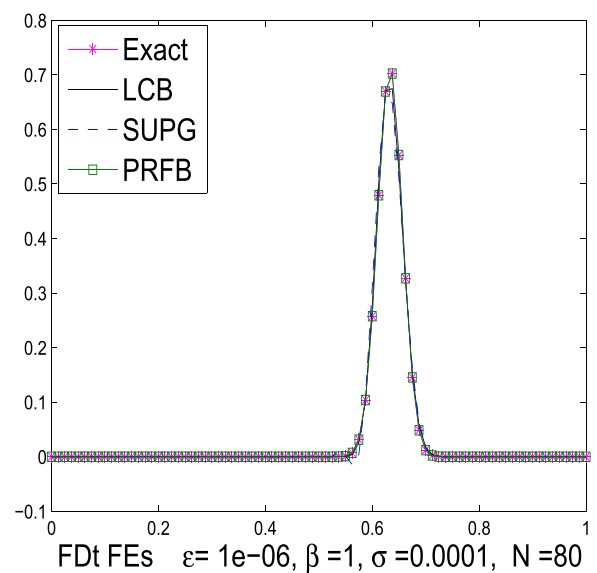

(a)

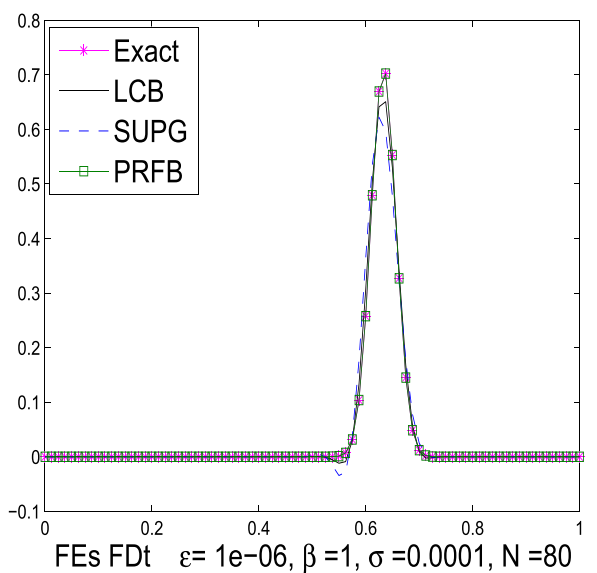

(b)

Figure 18. The numerical solutions for $\sigma=10^{-4}, \theta=1 / 2$, and $h=1 / 80$.

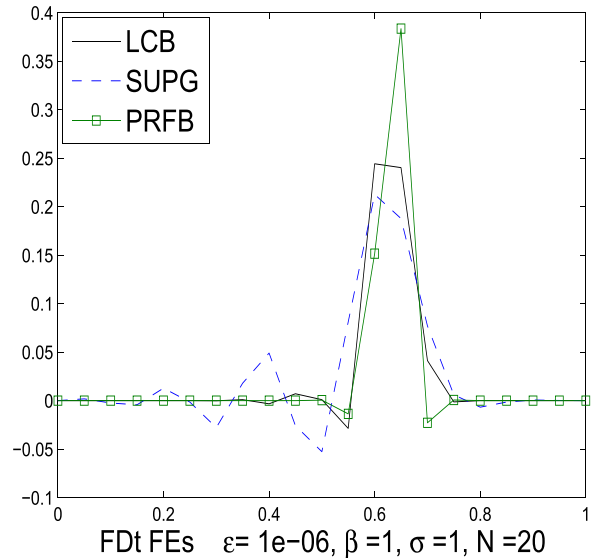

(a)

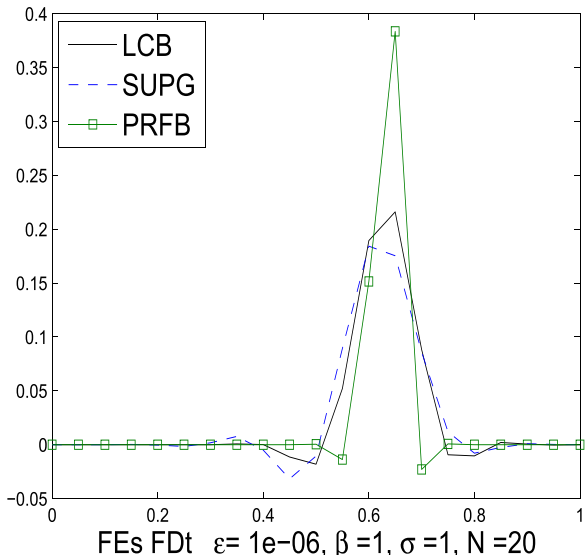

(b)

Figure 19. The numerical solutions for $\sigma=1, \theta=1 / 2$, and $h=1 / 20$. 


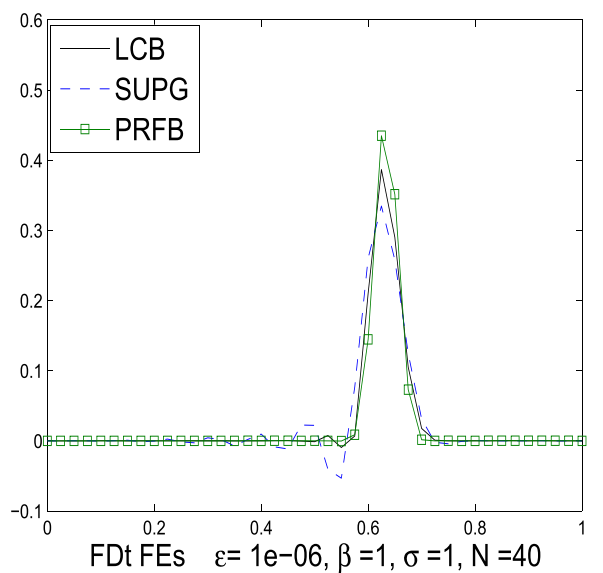

(a)

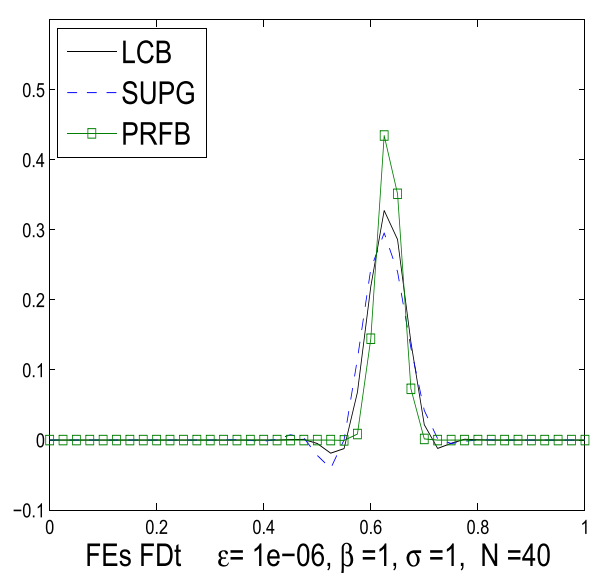

(b)

Figure 20. The numerical solutions for $\sigma=1, \theta=1 / 2$, and $h=1 / 40$.

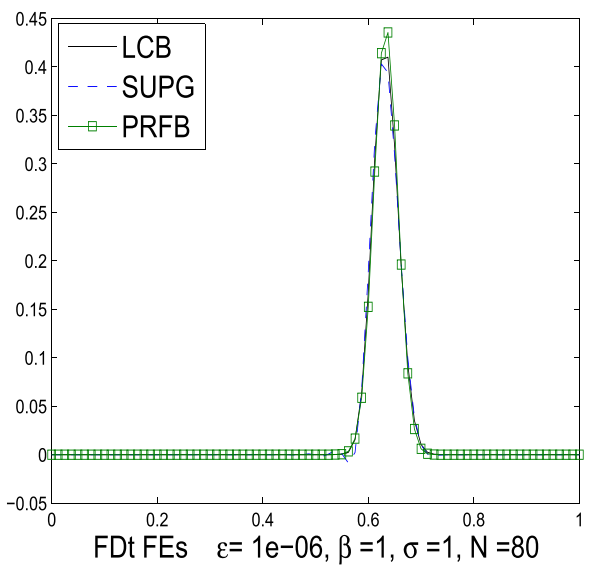

(a)

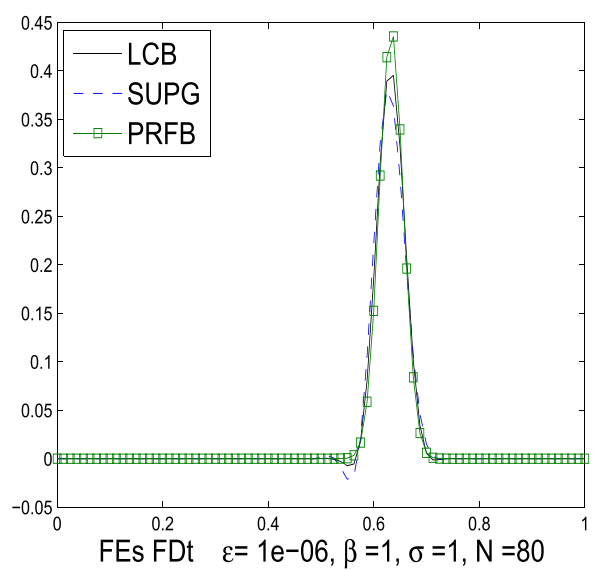

(b)

Figure 21. The numerical solutions for $\sigma=1, \theta=1 / 2$, and $h=1 / 80$.

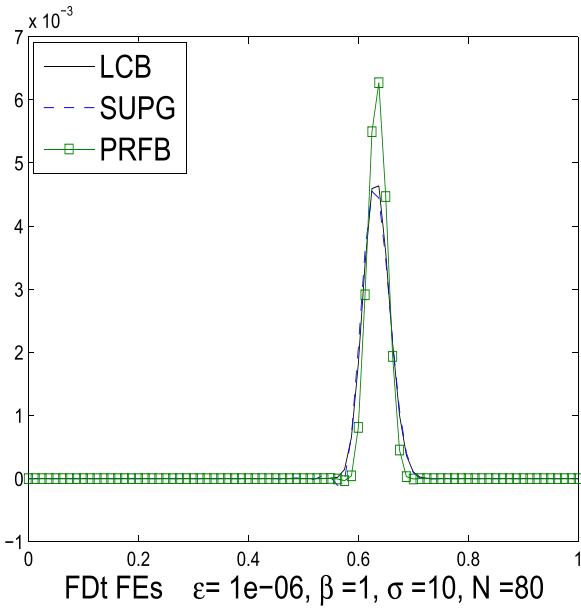

(a)

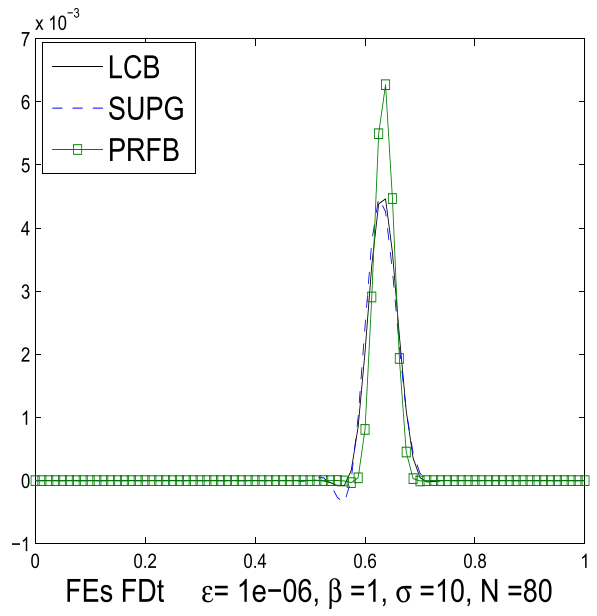

(b)

Figure 22. The numerical solutions for $\sigma=10, \theta=1 / 2$, and $h=1 / 80$. 


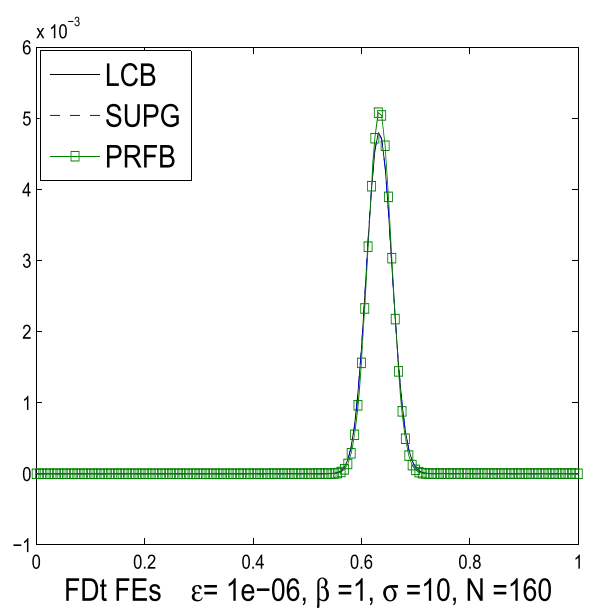

(a)

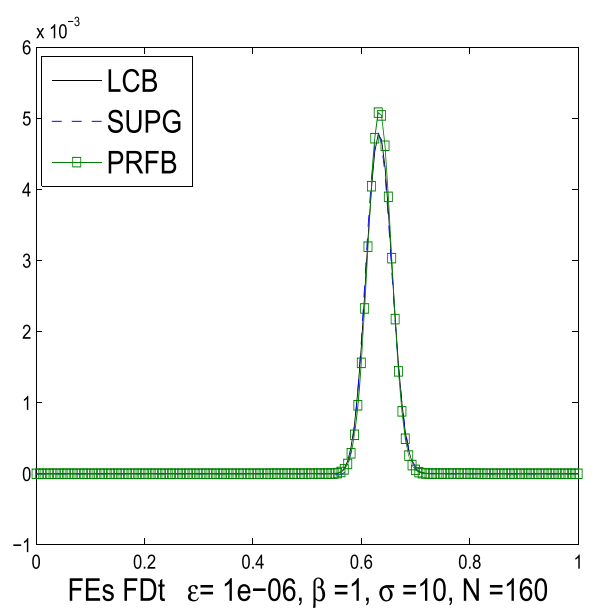

(b)

Figure 23. The numerical solutions for $\sigma=10, \theta=1 / 2$, and $h=1 / 160$.

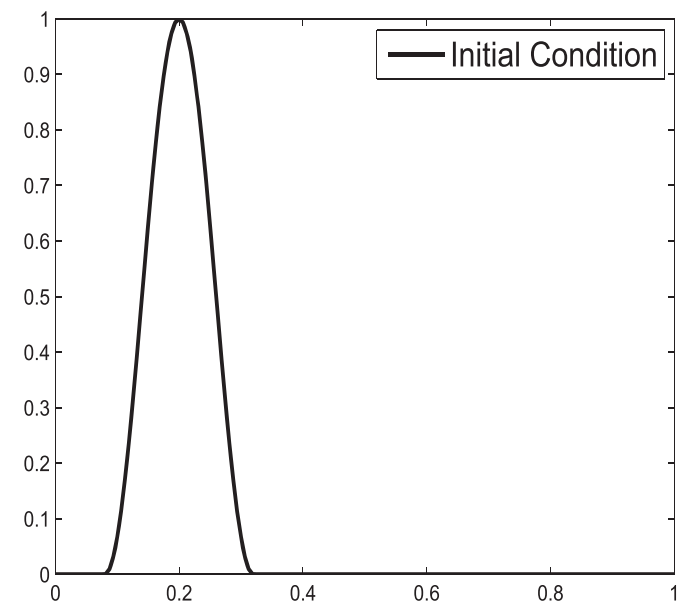

Figure 24. Cosine profile: initial condition.

The comparative study shows that the results obtained with the SUPG and LCB methods are more dissipative and dispersive in both $\mathrm{FD}_{t} \mathrm{FE}_{s}$ and $\mathrm{FE}_{s} \mathrm{FD}_{t}$ approaches, especially on coarse meshes, while the results show significant improvement with PRFB method.

5.0.4. Experiment 4: transport of a cosine profile. In this example, the propagation of the cosine function in Figure 24 at the initial profile is investigated [48], which is defined as

$$
u(x, 0)= \begin{cases}0.5\left(1+\cos \left(\pi \frac{x-0.2}{0.12}\right)\right), & \text { if }|x-0.2| \leqslant 0.12 \\ 0, & \text { otherwise }\end{cases}
$$

with the boundary condition as $u(0, t)=u(1, t)=0$. We take a uniform partition of $\Omega$ into subintervals of length $h=1 / 20,1 / 40,1 / 80,1 / 160$. In Figures 25-32, we set the value of the diffusion coefficient to be $\epsilon=10^{-6}$, the convective field $\beta=1$, the external force $f=0$, the final time $T=0.5$, and plot the solutions for different values of reaction $\sigma=10^{-4}, 1,10$.

The plots show that by combining the SUPG method with the $\mathrm{FE}_{s} \mathrm{FD}_{t}$ approach, the resulting approximate solution has less spurious oscillations than when it is combined with the $\mathrm{FD}_{t}$ $\mathrm{FE}_{s}$ approach. The LCB method performs better with $\mathrm{FD}_{t} \mathrm{FE}_{s}$ than with the other approach. 


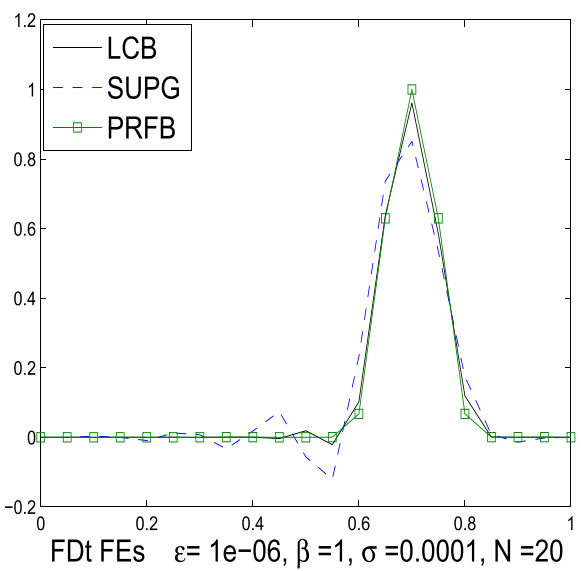

(a)

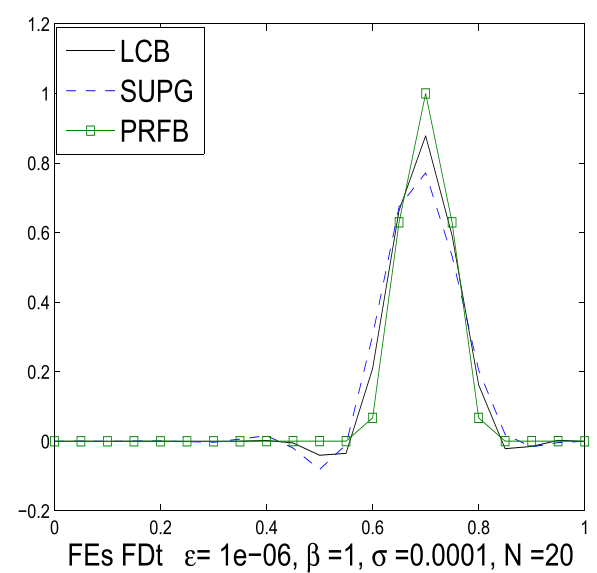

(b)

Figure 25. The numerical solutions for $\sigma=10^{-4}, \theta=1 / 2$, and $h=1 / 20$.

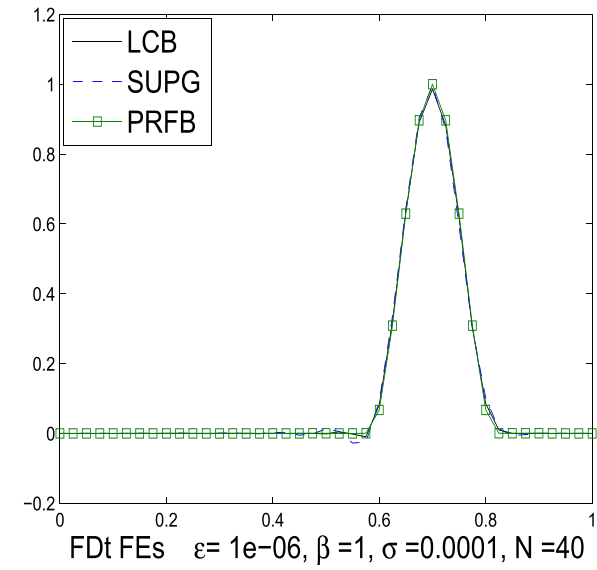

(a)

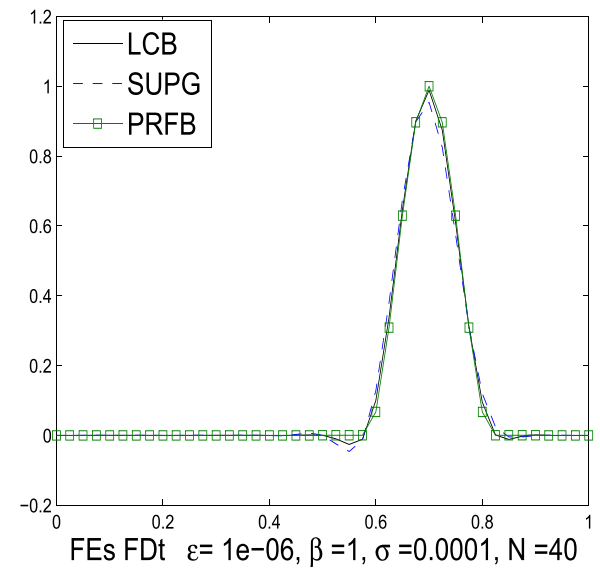

(b)

Figure 26. The numerical solutions for $\sigma=10^{-4}, \theta=1 / 2$, and $h=1 / 40$.

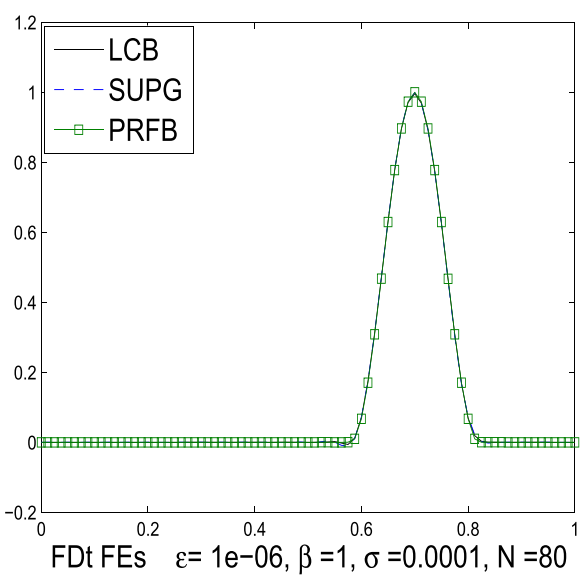

(a)

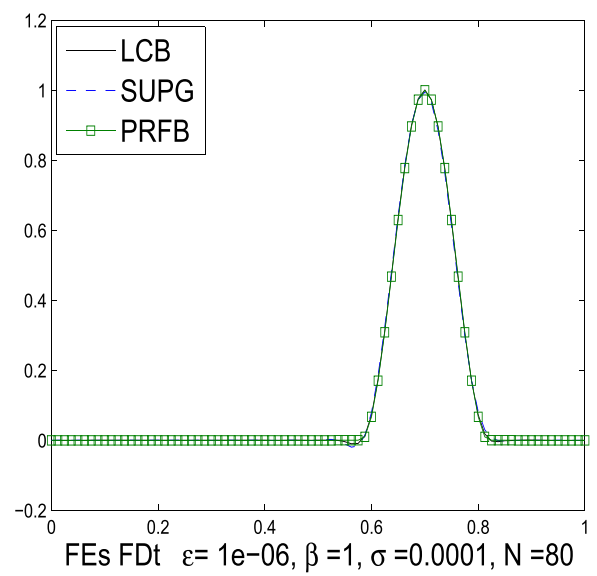

(b)

Figure 27. The numerical solutions for $\sigma=10^{-4}, \theta=1 / 2$, and $h=1 / 80$. 


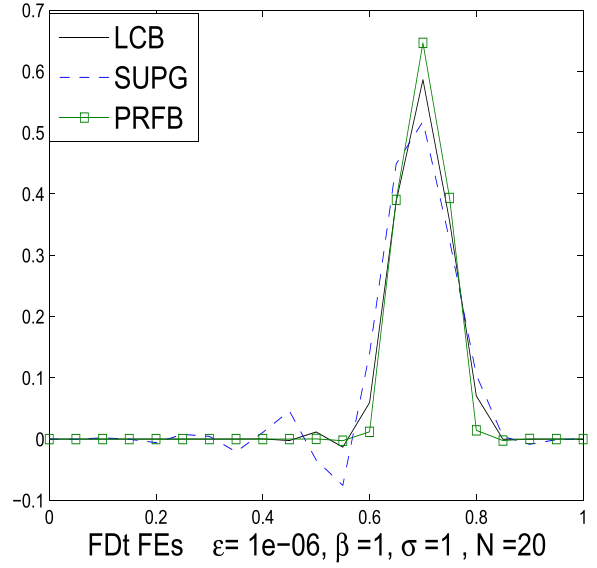

(a)

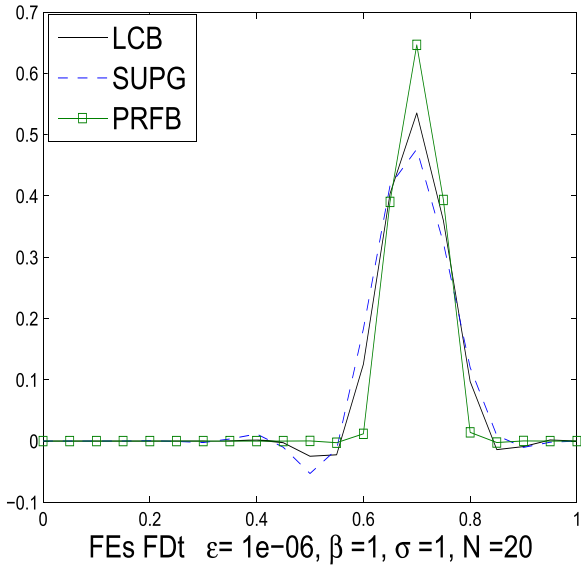

(b)

Figure 28. The numerical solutions for $\sigma=1, \theta=1 / 2$, and $h=1 / 20$.

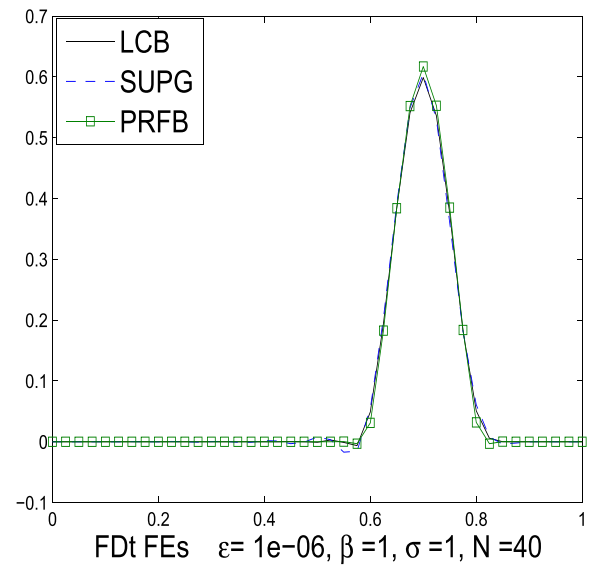

(a)

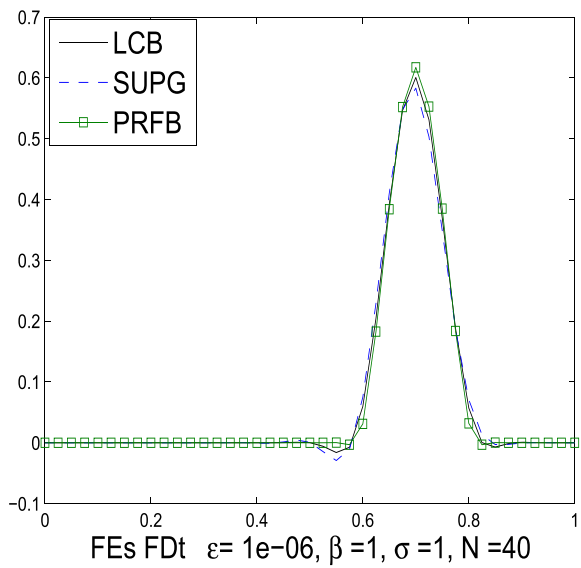

(b)

Figure 29. The numerical solutions for $\sigma=1, \theta=1 / 2$, and $h=1 / 40$.

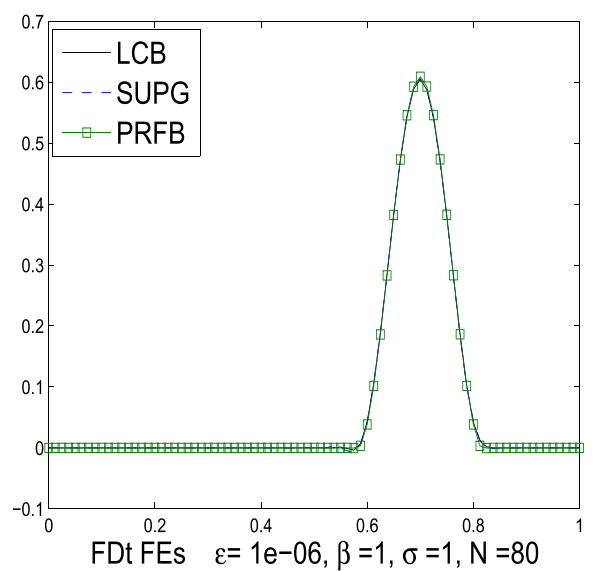

(a)

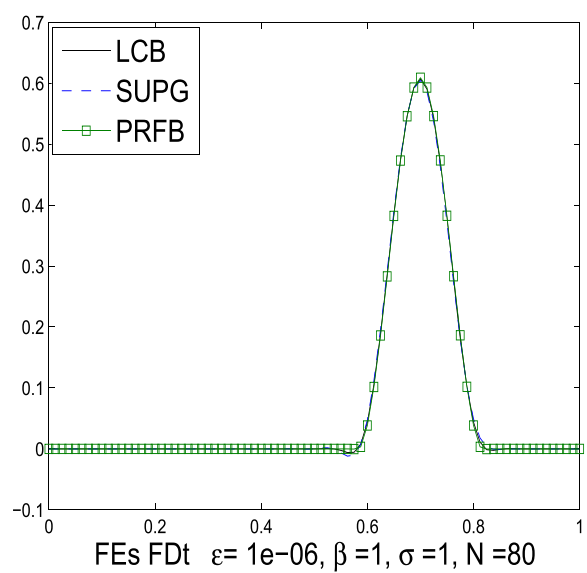

(b)

Figure 30. The numerical solutions for $\sigma=1, \theta=1 / 2$, and $h=1 / 80$. 


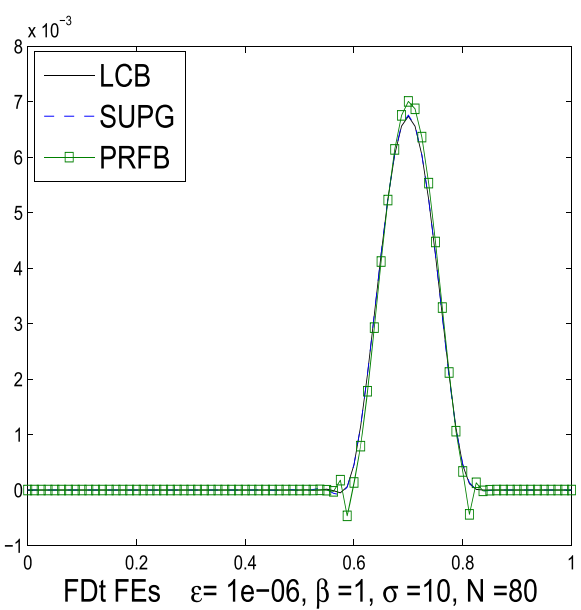

(a)

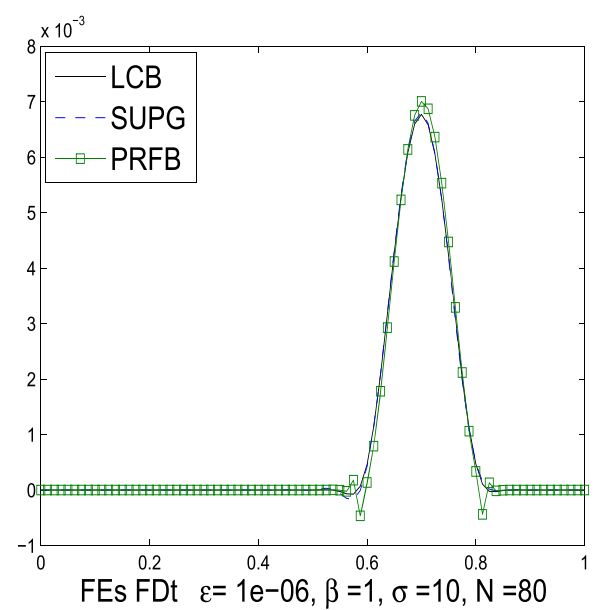

(b)

Figure 31. The numerical solutions for $\sigma=10, \theta=1 / 2$, and $h=1 / 80$.

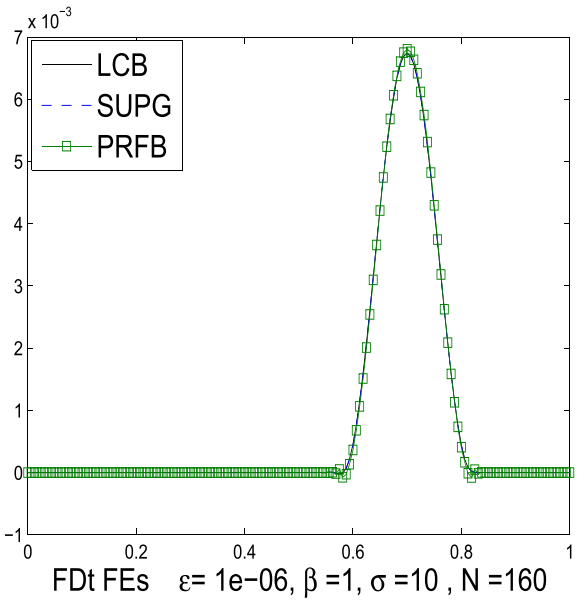

(a)

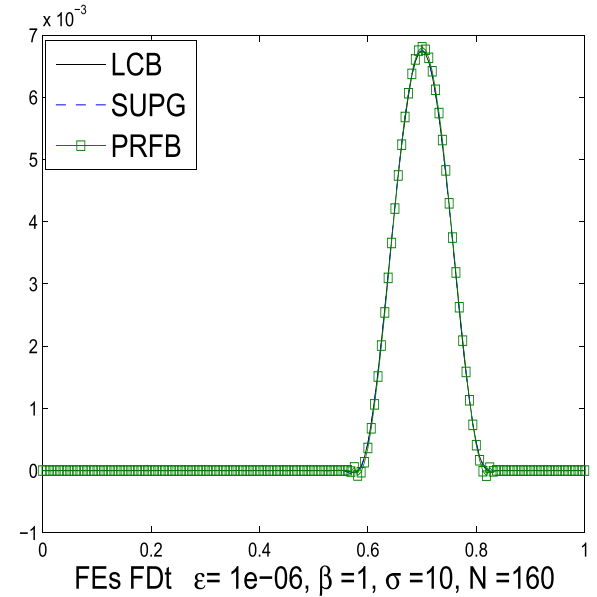

(b)

Figure 32. The numerical solutions for $\sigma=10, \theta=1 / 2$, and $h=1 / 160$.

However, results show significant improvement with the PRFB method in both $\mathrm{FD}_{t} \mathrm{FE}_{s}$ and $\mathrm{FE}_{s}$ $\mathrm{FD}_{t}$ approaches, especially on coarse meshes. We note that all the methods are equivalent when the mesh size is increased.

5.0.5. Experiment 5: transport of a square wave. The next example is the convection of a 1D square wave $[40,48]$. The initial condition is a unit square pulse presented in Figure 33 and can be defined as,

$$
u(x, 0)= \begin{cases}1, & \text { if }|x-0.3| \leqslant 0.1 \\ 0, & \text { otherwise }\end{cases}
$$

and the boundary condition as $u(0, t)=u(1, t)=0$. We take a uniform partition of $\Omega$ into subintervals of length $h=1 / 20,1 / 40,1 / 80$. In Figures 34-39, we set the value of the diffusion coefficient to be $\epsilon=10^{-6}$, the convective field $\beta=1$, the external force $f=0$, the final time $T=0.2$, and plot the solutions for different values of reaction $\sigma=10^{-4}, 1$.

The numerical solutions obtained with the SUPG method show that when using the $\mathrm{FE}_{s} \mathrm{FD}_{t}$ approach, the resulting approximate solution is less oscillatory than when it is combined with the 


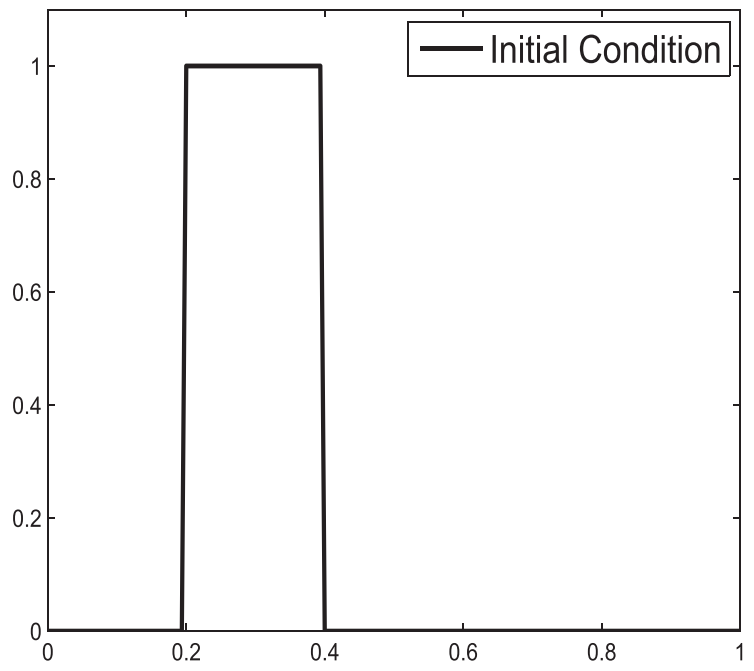

Figure 33. Transport of a square wave: initial condition.

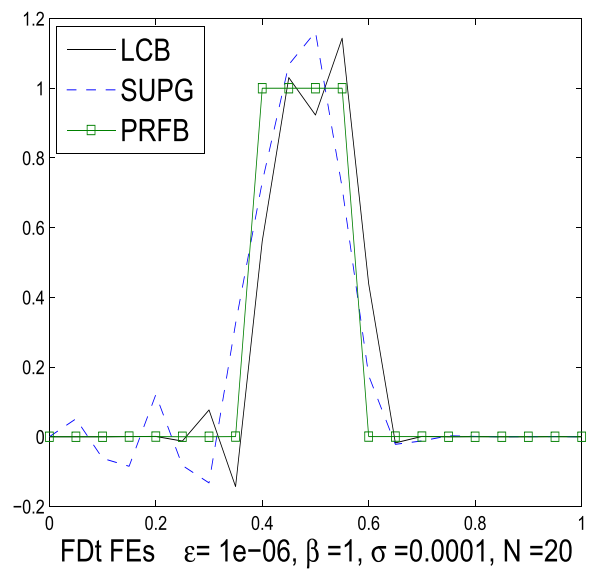

(a)

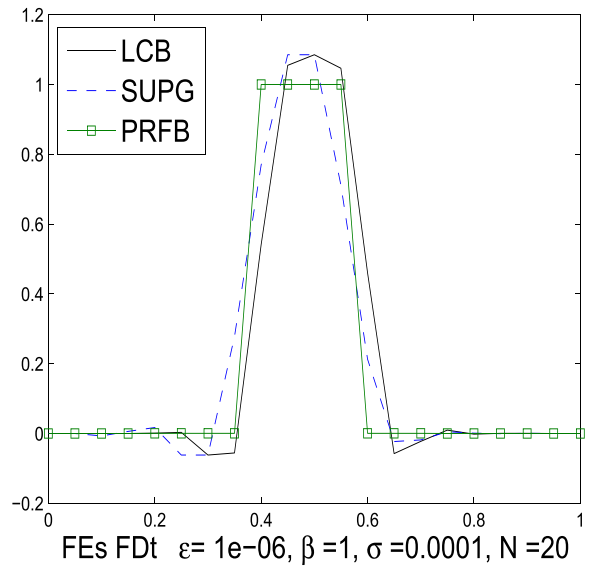

(b)

Figure 34. The numerical solutions for $\sigma=10^{-4}, \theta=1 / 2$, and $h=1 / 20$.

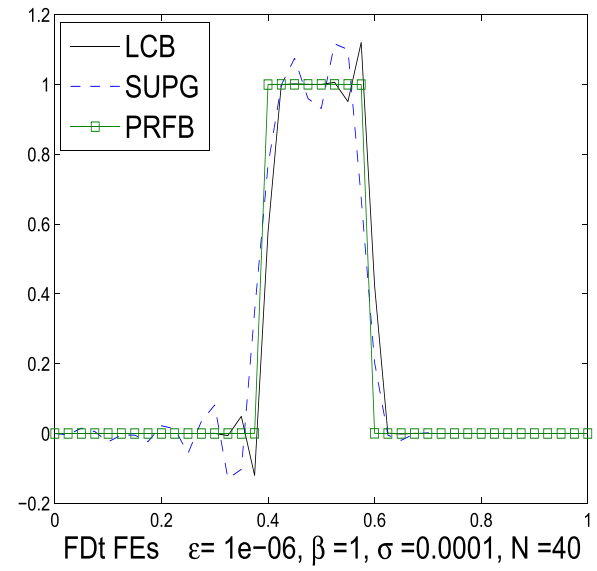

(a)

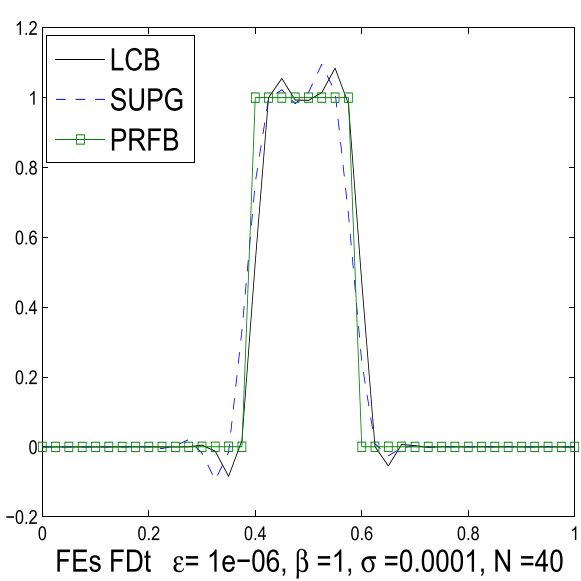

(b)

Figure 35. The numerical solutions for $\sigma=10^{-4}, \theta=1 / 2$, and $h=1 / 40$. 


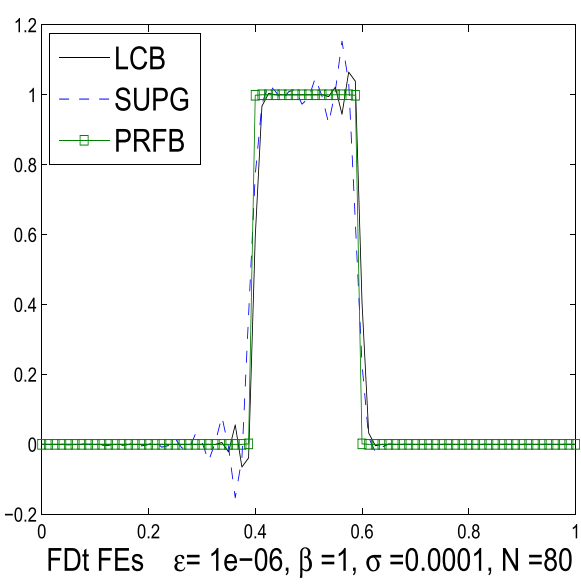

(a)

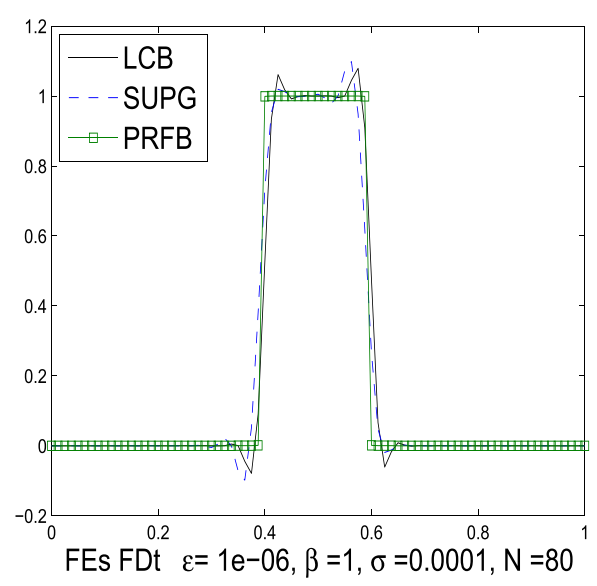

(b)

Figure 36. The numerical solutions for $\sigma=10^{-4}, \theta=1 / 2$, and $h=1 / 80$.

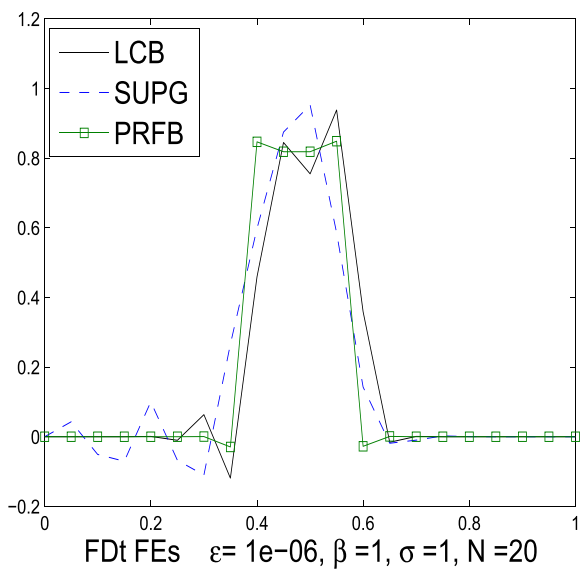

(a)

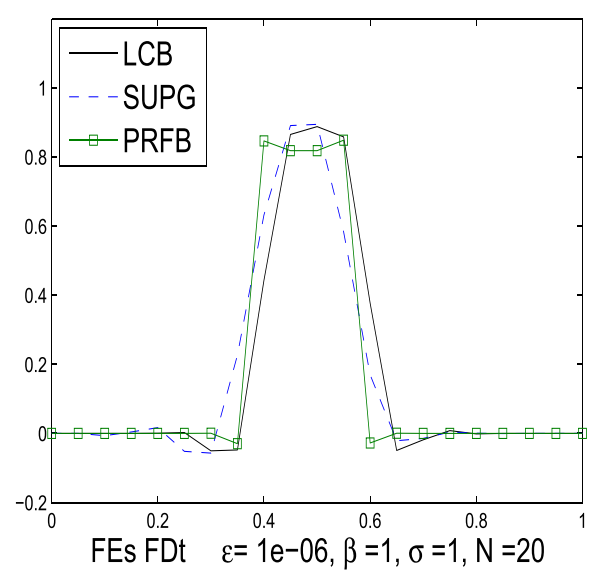

(b)

Figure 37. The numerical solutions for $\sigma=1, \theta=1 / 2$, and $h=1 / 20$.

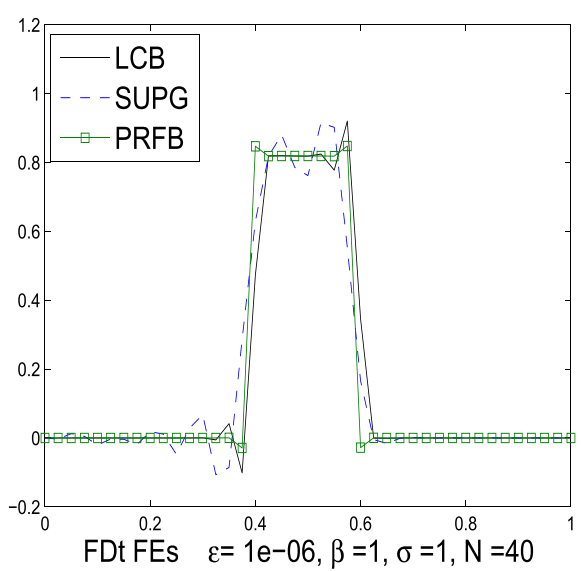

(a)

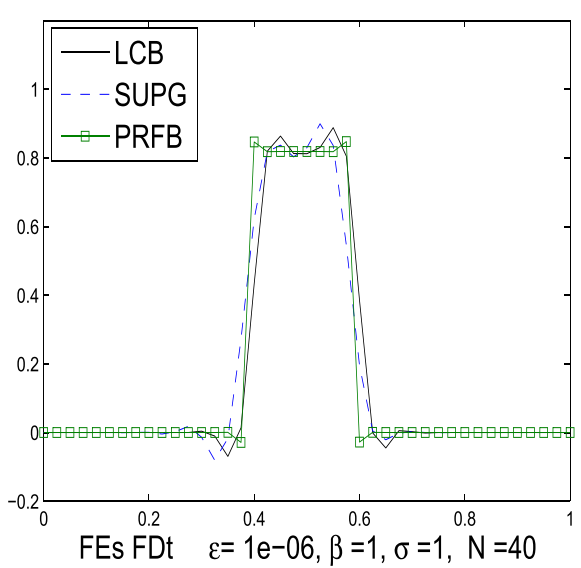

(b)

Figure 38. The numerical solutions for $\sigma=1, \theta=1 / 2$, and $h=1 / 40$. 


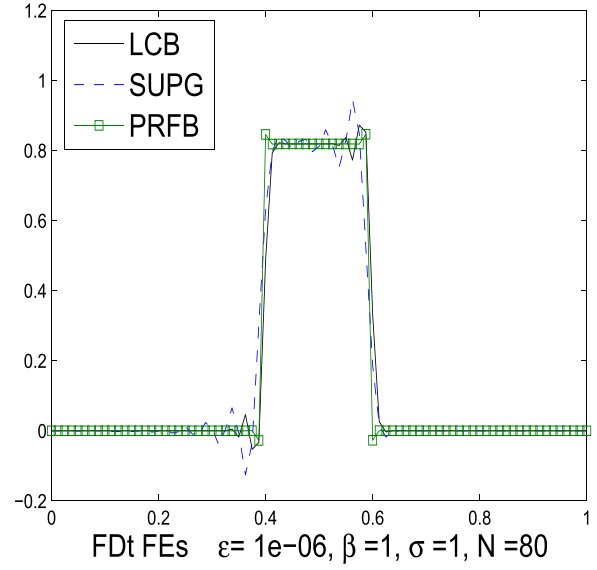

(a)

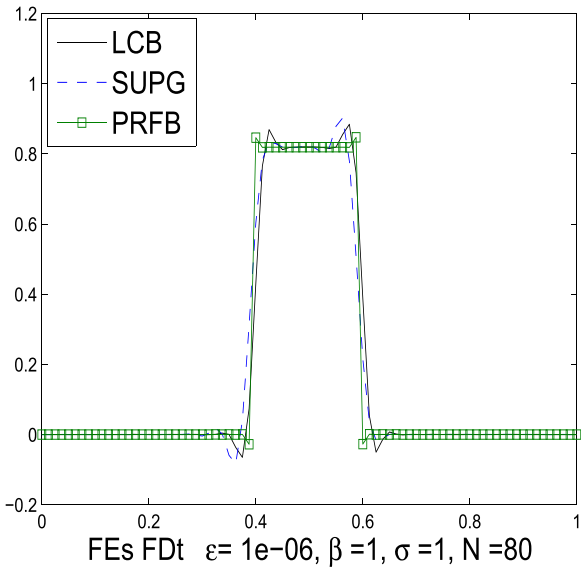

(b)

Figure 39. The numerical solutions for $\sigma=1, \theta=1 / 2$, and $h=1 / 80$.

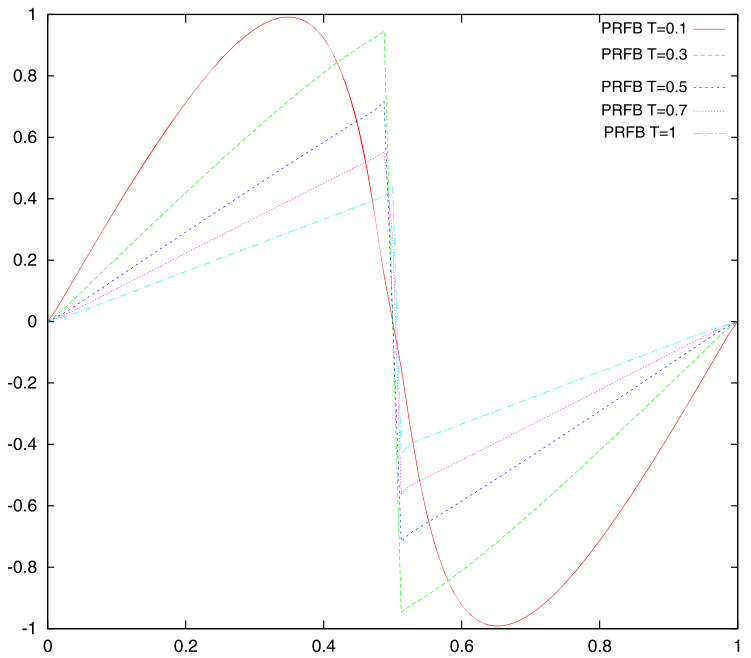

Figure 40. The numerical solution with pseudo residual-free bubbles (PRFB) method when $\epsilon=5 \times 10^{-5}$, $\theta=1 / 2$, and $h=1 / 80$ at different times.

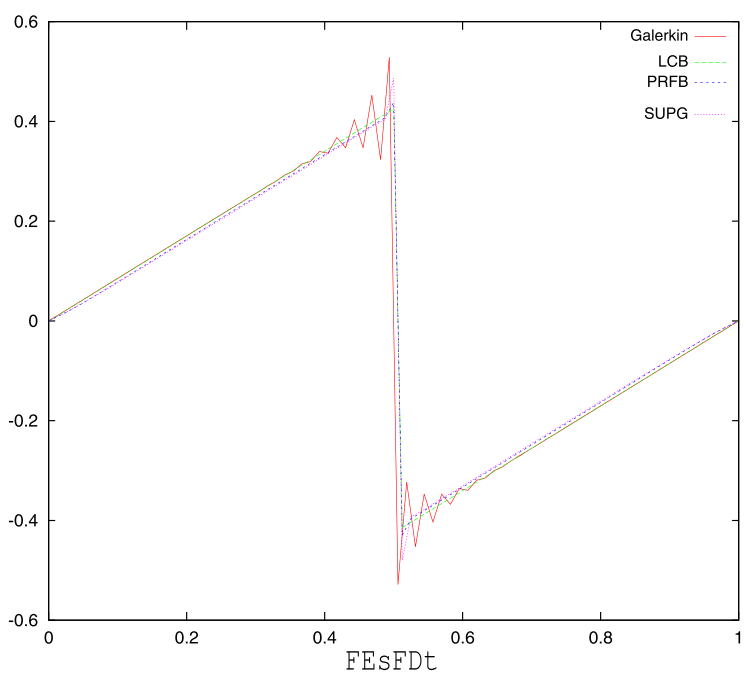

Figure 41. The numerical solutions for $\epsilon=5 \times 10^{-5}, T=1, \theta=1 / 2$, and $h=1 / 80$. 
$\mathrm{FD}_{t} \mathrm{FE}_{s}$ approach. With regards to the LCB strategy, the spurious oscillations are present when using $\mathrm{FE}_{s} \mathrm{FD}_{t}$, while with the $\mathrm{FD}_{t} \mathrm{FE}_{s}$ approach, they have almost disappeared. However, the PRFB method works well in both $\mathrm{FD}_{t} \mathrm{FE}_{s}$ and $\mathrm{FE}_{s} \mathrm{FD}_{t}$ approaches even on coarse meshes.

5.0.6. Experiment 6: Burgers' equation. Finally, we consider the numerical approximation of the following problem,

$$
\left\{\begin{array}{l}
u_{t}-\epsilon u_{x x}+u u_{x}=0, \quad x \in(0,1), 0 \leqslant t \leqslant T \\
u(0, t)=u(1, t)=0, \quad t \in(0, T) \\
u(x, 0)=\sin (2 \pi x), \quad 0<x<1
\end{array}\right.
$$

We take a uniform partition of $\Omega$ into subintervals of length $h=1 / 80$. In Figure 40, we set the value of the diffusion coefficient to be $\epsilon=5 \times 10^{-5}$, and plot the solutions at different times $T=0.1,0.3,0.5,0.7,1$. Figure 41 displays the solutions obtained with the vertical method of lines

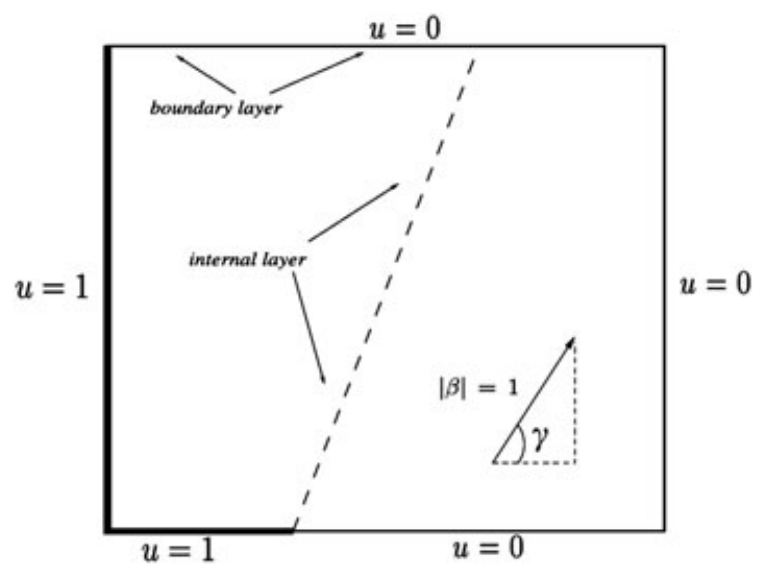

Figure 42. Configuration of Experiment 7.

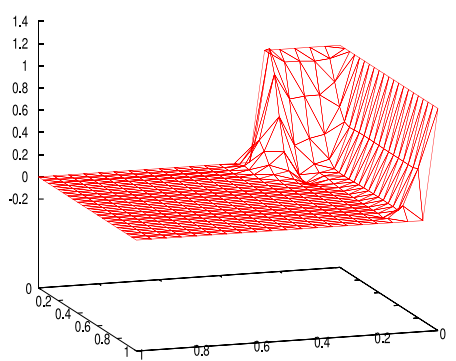

(a)

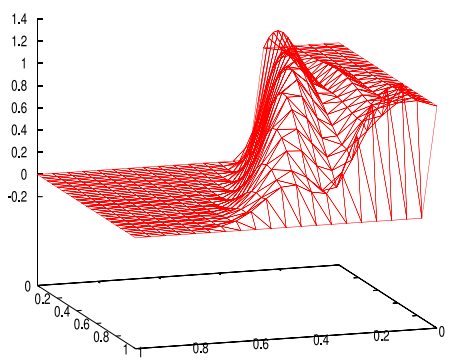

(d)

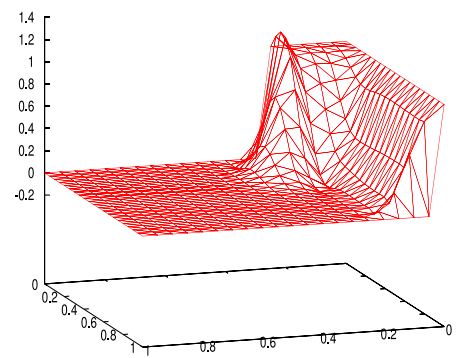

(b)

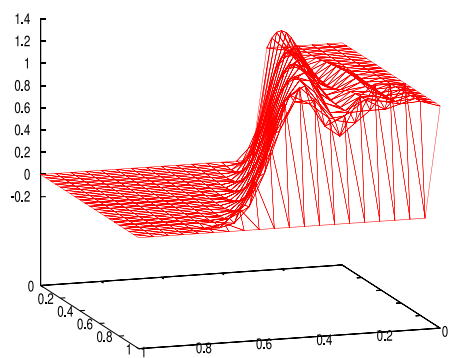

(e)

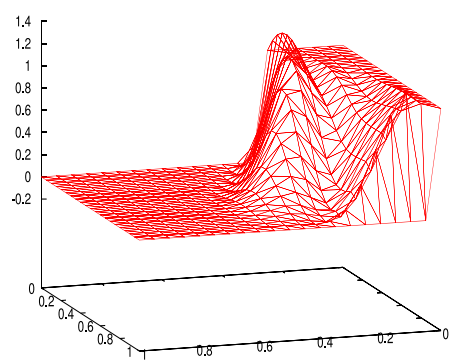

(c)

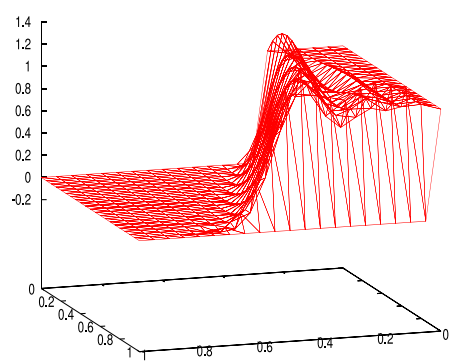

(f)

Figure 43. The numerical solution with the pseudo residual-free bubbles method when $\epsilon=10^{-4}, \sigma=f=$ 0.001 , and $\theta=1$ at different time steps. 
where standard Galerkin, SUPG, LCB, and PRFB methods are used in the spatial discretizations. The approximations generated by the SUPG method possess spurious oscillations in layer regions, while the numerical solutions obtained with the PRFB and LCB methods capture the position of the layers in general quite well.

5.0.7. Experiment 7. Finally, we perform a numerical test in $2 \mathrm{D}$ case, in which the problem domain is the unit square, that is, $\Omega=[0,1] \times[0,1]$. We consider a decomposition of $\Omega$ into a set of triangles. The choice of subgrid nodes in a typical triangular element is technically similar to the 1D case, and we refer to [42] for the details. The boundary conditions are displayed in Figure 42, and the initial condition is defined as $u(x, y, 0)=0$. We take a set of uniform triangular mesh, which is made up of 20 elements, respectively, in $x$ and $y$ directions, and we set $\epsilon=10^{-4}, \gamma=72^{0}, \sigma=f=0.001$, the final time $T=1.4$. Figure 43 displays the solutions obtained with the vertical method of lines where PRFB method [42] is used in the spatial discretizations. The results show the potential of the proposed method for $2 \mathrm{D}$ problems.

\section{CONCLUSION}

The PRFB method is proposed for the approximate solution of time-dependent convectiondiffusion-reaction Equation (1), and it is compared with two different stabilized methods on several benchmark problems. Numerical experiments cover a variety of problem configurations, and the results illustrate the good performance of the PRFB method even on coarse meshes as compared with the others. The improved approximations over the SUPG and LCB methods were especially obtained around layers, in which overshoots were significantly diminished through the PRFB method. The application of the PRFB method to further examples underlines potential of the proposed approach for the nonlinear and 2D problems.

\section{REFERENCES}

1. Asensio MI, Ayuso B, Ferragut L, Sangalli G. Numerical methods for modelling leaching of pollutants in soils. Advances in Engineering Software 2007; 38:429-438.

2. Bazilevs Y, Calo VM, Tezduyar TE, Hughes TJR. YZ $\beta$ discontinuity-capturing for advection-dominated processes with application to arterial drug delivery. International Journal for Numerical Methods in Fluids 2007; 54:593-608.

3. Codina R, Principe J, Munoz C, Baiges J. Numerical modelling of chlorine concentration in water storage tanks. International Journal for Numerical Methods in Fluids 2015; 79:84-107.

4. John V, Mitkova T, Roland M, Sundmacher K, Tobiska L, Voigt A. Simulations of population balance systems with one internal coordinate using finite element methods. Chemical Engineering Science 2009; 64:733-741.

5. Murray JD. Mathematical Biology II: Spatial Models and Biomedical Applications, Interdisciplinary Applied Mathematics, vol. 18. Springer: New York, 2003.

6. Roose T, Chapman SJ, Maini PK. Mathematical models of avascular tumor growth. SIAM Review 2007; 49:179-208.

7. Frutos J, Garcia AB, Novo J. An adaptive SUPG method for evolutionary convection-diffusion equations. Computer Methods in Applied Mechanics and Engineering 2014; 273:219-237.

8. Frutos J, Garcia AB, Novo J. Local error estimates for the SUPG method applied to evolutionary convectionreaction-diffusion equations. Journal of Scientific Computing 2016; 66:528-554.

9. Franca LP, Ramalho JVA, Valentin F. Enriched finite element methods for unsteady reaction-diffusion problems. Communications in Numerical Methods in Engineering 2006; 22:619-625.

10. Hundsdorfer W, Verwer JG. Numerical Solution of Time-dependent Advection-Diffusion-Reaction Equations. Springer: Berlin, 2003.

11. John V, Novo J. On (essentially) non-oscillatory discretizations of evolutionary convection-diffusion equations. Journal of Computational Physics 2012; 231:1570-1585.

12. Asensio MI, Ayuso B, Sangalli G. Time-space \& space-time elements for unsteady advection dominated problems. In Numerical Mathematics and Advances Applications, Springer, Berlin Heidelberg, 2006; 328-335.

13. Codina R, Blasco J. Analysis of a stabilized finite element approximation of the transient convection-diffusionreaction equation using orthogonal subscales. Computing and Visualization in Science 2002; 4:167-174.

14. Douglas J, Russell TF. Numerical methods for convection-dominated diffusion problems based on combining the method of characteristics with finite element or finite difference procedures. SIAM Journal on Numerical Analysis 1982; 19:871-885.

15. Hauke G, Doweidar MH. Fourier analysis of semidiscrete and space-time stabilized methods for the advectivereactive diffusive equation: I. SUPG. Computer Methods in Applied Mechanics and Engineering 2005; 194: $45-81$. 
16. Hauke G, Doweidar MH. Fourier analysis of semidiscrete and space-time stabilized methods for the advectivereactive-diffusive equation: II. SGS. Computer Methods in Applied Mechanics and Engineering 2005; 194: 691-725.

17. Huerta A, Donea J. Time-accurate solution of stabilized convection-diffusion-reaction equations: I, time and space discretization. Communications in Numerical Methods in Engineering 2002; 18:565-573.

18. Masud A, Hughes TJR. A space-time Galerkin/least-squares finite element formulation of the Navier-Stokes equations for moving domain problems. Computer Methods in Applied Mechanics and Engineering 1997; 146: 91-126.

19. Tezduyar TE, Ganjoo DK. Petrov-Galerkin formulations with weighting functions dependent upon spatial and temporal discretization: applications to transient convection-diffusion problems. Computer Methods in Applied Mechanics and Engineering 1986; 59:49-71.

20. Tezduyar TE, Park YJ, Deans HA. Finite element procedures for time-dependent convection-diffusion-reaction systems. International Journal for Numerical Methods in Fluids 1987; 7:1013-1033.

21. Tezduyar TE, Sathe S. Enhanced-discretization space-time technique (EDSTT). Computer Methods in Applied Mechanics and Engineering 2004; 193:1385-1401.

22. Thomee V. Galerkin Finite Element Methods for Parabolic Problems, Vol. 25. Springer-Verlag: Berlin, 1997.

23. Bochev PB, Gunzburger MD, Shadid JN. Stability of the SUPG finite element method for transient advectiondiffusion problems. Computer Methods in Applied Mechanics and Engineering 2004; 193:2301-2323.

24. John V, Novo J. Error analysis of the SUPG finite element discretization of evolutionary convection-diffusionreaction equations. SIAM Journal on Numerical Analysis 2011; 49:1149-1176.

25. Lube G, Weiss D. Stabilized finite element methods for singularly perturbed parabolic problems. Applied Numerical Mathematics 1995; 17:431-459.

26. Almeida RC, Silva RS. A stable Petrov-Galerkin method for convection-dominated problems. Computer Methods in Applied Mechanics and Engineering 1997; 140:291-304.

27. Hughes TJR. Recent progress in the development and understanding of SUPG methods with special reference to the compressible Euler and Navier-Stokes equations. International Journal for Numerical Methods in Fluids 1987; 7:1261-1275.

28. John V, Knobloch P. On spurious oscillations at layers diminishing (SOLD) methods for convection-diffusion equations: Part I - A review. Computer Methods in Applied Mechanics and Engineering 2007; 196:2197-2215.

29. Brezzi F, Bristeau MO, Franca LP, Mallet M, Roge G. A relationship between stabilized finite element methods and the Galerkin method with bubble functions. Computer Methods in Applied Mechanics and Engineering 1992; 96:117-129.

30. Brezzi F, Russo A. Choosing bubbles for advection-diffusion problems. Mathematical Models and Methods in Applied Sciences 1994; 4:571-587.

31. Franca LP, Farhat C. Bubble functions prompt unusual stabilized finite element methods. Computer Methods in Applied Mechanics and Engineering 1995; 123:299-308.

32. Franca LP, Farhat C, Lesoinne M, Russo A. Unusual stabilized finite element methods and residual free bubbles. International Journal for Numerical Methods in Fluids 1998; 27:159-168.

33. Frutos J, Novo J. Bubble stabilization of linear finite element methods for nonlinear evolutionary convectiondiffusion equations. Computer Methods in Applied Mechanics and Engineering 2008; 197:3988-3999.

34. Hughes TJR. Multiscale phenomena: Green's functions, the Dirichlet-to-Neumann formulation, subgrid scale models, bubbles and the origin of stabilized methods. Computer Methods in Applied Mechanics and Engineering 1995; 127:387-401.

35. Brezzi F, Marini D, Russo A. On the choice of a stabilizing sub-grid for convection-diffusion problems. Computer Methods in Applied Mechanics and Engineering 2005; 194:127-148.

36. Brezzi F, Marini D, Russo A. Applications of pseudo residual-free bubbles to the stabilization of convectiondiffusion problems. Computer Methods in Applied Mechanics and Engineering 1998; 166:51-63.

37. Nesliturk A. A stabilizing sub-grid for convection-diffusion problem. M3AS 2006; 16:211-231.

38. Nesliturk AI. On the choice of stabilizing subgrid for convection-diffusion problem on rectangular grids. Computers \& Mathematics with Applications 2010; 59:3687-3699.

39. Brezzi F, Hauke G, Marini D, Sangalli G. Link-cutting bubbles for the stabilization of convection-diffusion-reaction problems. Mathematical Models and Methods in Applied Sciences 2003; 13:445-461.

40. Asensio MI, Ayuso B, Sangalli G. Coupling stabilized finite element methods with finite difference time integration for advection-diffusion-reaction problems. Computer Methods in Applied Mechanics and Engineering 2007; 196:3475-3491.

41. Sendur A, Nesliturk AI. Applications of the pseudo residual-free bubbles to the stabilization of-diffusion-reaction problems. Calcolo 2012; 49:1-19.

42. Sendur A, Nesliturk AI, Kaya A. Applications of RFBs to the stabilization of the convection-diffusion-reaction problems in 2D. Computer Methods in Applied Mechanics and Engineering 2014; 277:154-179.

43. Grossmann C, Roos HG. Numerik Partieller Differentialgleichungen. Springer Vieweg Verlag: Stuttgart, 1992.

44. Codina R. Comparison of some finite element methods for solving the diffusion-convection-reaction equation. Computer Methods in Applied Mechanics and Engineering 1998; 156:185-210.

45. John V, Schmeyer E. Finite element methods for time-dependent convection-diffusion-reaction equations with small diffusion. Computer Methods in Applied Mechanics and Engineering 2008; 198:475-494. 
46. Harari I. Stability of semidiscrete formulations for parabolic problems at small time steps. Computer Methods in Applied Mechanics and Engineering 2004; 193:1491-1516.

47. Harari I, Hauke G. Semidiscrete formulations for transient transport at small time steps. International Journal for Numerical Methods in Fluids 2007; 54:731-743.

48. Henao CAA, Coutinho ALGA, Franca LP. A stabilized method for transient transport equations. Computational Mechanics 2010; 46:199-204.

49. Raviart PA, Thomas JM. Introduction al Analyse Numerique des Equations aux Derivees Partielles. Masson: Paris, 1992.

50. Franca LP, Frey SL, Hughes TJR. Stabilized finite element methods: I. Application to the advective-diffusive model. Computer Methods in Applied Mechanics and Engineering 1992; 95:253-276.

51. Catabriga L, Coutinho ALGA, Tezduyar TE. Compressible flow SUPG parameters computed from element matrices. Communications in Numerical Methods in Engineering 2005; 21:465-476.

52. Tezduyar TE, Sathe S. Stabilization parameters in SUPG and PSPG formulations. Journal of Computational and Applied Mechanics 2003; 4:71-88.

53. Asensio MI, Ayuso B, Sangalli G. Bubbles for the stabilization of transient convection-diffusion-reaction problems. Proceedings of the European Congress on Computational Methods in Applied Sciences and Engineering, ECCOMAS, Jyvaskyla, Finland, July 2004; 24-28.

54. Kaya A. Pseudo residual-free bubble functions for the stabilization of convection-diffusion-reaction problems. M.Sc. Thesis, Izmir Institute of Technology, 2012.

55. Donea J, Huerta A. Finite Element Methods for Flow Problems. John Wiley and Sons: Chichester, UK, 2003. 